\title{
$\mathfrak{s l}(2)$-Trivial Deformations of $\operatorname{Vect}_{\mathrm{Pol}}(\mathbb{R})$-Modules of Symbols ${ }^{\star}$
}

Mabrouk BEN AMMAR and Maha BOUJELBENE

Département de Mathématiques, Faculté des Sciences de Sfax, BP 802, 3038 Sfax, Tunisie

E-mail: mabrouk.benammar@fss.rnu.tn,maha.boujelben@fss.rnu.tn

Received January 14, 2008, in final form September 05, 2008; Published online September 18, 2008

Original article is available at http://www.emis.de/journals/SIGMA/2008/065/

\begin{abstract}
We consider the action of $\operatorname{Vect}_{\mathrm{Pol}}(\mathbb{R})$ by Lie derivative on the spaces of symbols of differential operators. We study the deformations of this action that become trivial once restricted to $\mathfrak{s l}(2)$. Necessary and sufficient conditions for integrability of infinitesimal deformations are given.
\end{abstract}

Key words: tensor densities, cohomology, deformations

2000 Mathematics Subject Classification: 17B56; 17B66; 53D55

\section{Introduction}

Let $\operatorname{Vect}_{\mathrm{Pol}}(\mathbb{R})$ be the Lie algebra of polynomial vector fields on $\mathbb{R}$. Consider the 1-parameter action of $\operatorname{Vect}_{\mathrm{Pol}}(\mathbb{R})$ on the space $\mathbb{R}[x]$ of polynomial functions on $\mathbb{R}$ defined by

$$
L_{X \frac{d}{d x}}^{\lambda}(f)=X f^{\prime}+\lambda X^{\prime} f
$$

where $X, f \in \mathbb{R}[x]$ and $X^{\prime}:=\frac{d X}{d x}$. Denote by $\mathcal{F}_{\lambda}$ the $\operatorname{Vect}_{\mathrm{Pol}}(\mathbb{R})$-module structure on $\mathbb{R}[x]$ defined by this action for a fixed $\lambda$. Geometrically, $\mathcal{F}_{\lambda}$ is the space of polynomial weighted densities of weight $\lambda$ on $\mathbb{R}$

$$
\mathcal{F}_{\lambda}=\left\{f d x^{\lambda} \mid f \in \mathbb{R}[x]\right\} .
$$

The space $\mathcal{F}_{\lambda}$ coincides with the space of vector fields, functions and differential 1-forms for $\lambda=-1,0$ and 1 , respectively.

Denote by $\mathcal{D}_{\nu, \mu}:=\operatorname{Hom}_{\text {diff }}\left(\mathcal{F}_{\nu}, \mathcal{F}_{\mu}\right)$ the $\operatorname{Vect}_{\text {Pol }}(\mathbb{R})$-module of linear differential operators with the $\operatorname{Vect}_{\mathrm{Pol}}(\mathbb{R})$-action given by the formula

$$
L_{X}^{\nu, \mu}(A)=L_{X}^{\mu} \circ A-A \circ L_{X}^{\nu}
$$

Each module $\mathcal{D}_{\nu, \mu}$ has a natural filtration by the order of differential operators; the graded module $\mathcal{S}_{\nu, \mu}:=\operatorname{gr} \mathcal{D}_{\nu, \mu}$ is called the space of symbols. The quotient-module $\mathcal{D}_{\nu, \mu}^{k} / \mathcal{D}_{\nu, \mu}^{k-1}$ is isomorphic to the module of tensor densities $\mathcal{F}_{\mu-\nu-k}$, the isomorphism is provided by the principal symbol $\sigma$ defined by

$$
A=\sum_{i=0}^{k} a_{i}(x) \partial_{x}^{i} \mapsto \sigma(A)=a_{k}(x)(d x)^{\mu-\nu-k}
$$

\footnotetext{
${ }^{\star}$ This paper is a contribution to the Special Issue on Deformation Quantization. The full collection is available at http://www.emis.de/journals/SIGMA/Deformation_Quantization.html
} 
(see, e.g., [10]). As a $\operatorname{Vect}_{\mathrm{Pol}}(\mathbb{R})$-module, the space $\mathcal{S}_{\nu, \mu}$ depends only on the difference $\delta=\mu-\nu$, so that $\mathcal{S}_{\nu, \mu}$ can be written as $\mathcal{S}_{\delta}$, and we have

$$
\mathcal{S}_{\delta}=\bigoplus_{k=0}^{\infty} \mathcal{F}_{\delta-k}
$$

as $\operatorname{Vect}_{\mathrm{Pol}}(\mathbb{R})$-modules. The space of symbols of order $\leq n$ is

$$
\mathcal{S}_{\delta}^{n}:=\bigoplus_{j=0}^{n} \mathcal{F}_{\delta-j} .
$$

The space $\mathcal{D}_{\nu, \mu}$ cannot be isomorphic as a $\operatorname{Vect}_{\text {Pol }}(\mathbb{R})$-module to the corresponding space of symbols, but is a deformation of this space in the sense of Richardson-Neijenhuis [12]; however, they are isomorphic as $\mathfrak{s l}(2)$-modules (see [9]). In the last two decades, deformations of various types of structures have assumed an ever increasing role in mathematics and physics. For each such deformation problem a goal is to determine if all related deformation obstructions vanish and many beautiful techniques had been developed to determine when this is so. Deformations of Lie algebras with base and versal deformations were already considered by Fialowski [5]. It was further developed, with introduction of a complete local algebra base (local means a commutative algebra which has a unique maximal ideal) by Fialowski [6]. Also, in [6], the notion of miniversal (or formal versal) deformation was introduced in general, and it was proved that under some cohomology restrictions, a versal deformation exists. Later Fialowski and Fuchs, using this framework, gave a construction for the versal deformation [7].

We use the framework of Fialowski [6] (see also [1] and [2]) and consider (multi-parameter) deformations over complete local algebras. We construct the miniversal deformation of this action and define the complete local algebra related to this deformation.

According to Nijenhuis-Richardson [12], deformation theory of modules is closely related to the computation of cohomology. More precisely, given a Lie algebra $\mathfrak{g}$ and a $\mathfrak{g}$-module $V$, the infinitesimal deformations of the $\mathfrak{g}$-module structure on $V$, i.e., deformations that are linear in the parameter of deformation, are related to $\mathrm{H}^{1}(\mathfrak{g} ; \operatorname{End}(V))$. The obstructions to extension of any infinitesimal deformation to a formal one are related to $\mathrm{H}^{2}(\mathfrak{g} ; \operatorname{End}(V))$. More generally, if $\mathfrak{h}$ is a subalgebra of $\mathfrak{g}$, then the $\mathfrak{h}$-relative cohomology space $\mathrm{H}^{1}(\mathfrak{g}, \mathfrak{h}$; $\operatorname{End}(V))$ measures the infinitesimal deformations that become trivial once the action is restricted to $\mathfrak{h}(\mathfrak{h}$-trivial deformations), while the obstructions to extension of any $\mathfrak{h}$-trivial infinitesimal deformation to a formal one are related to $\mathrm{H}^{2}(\mathfrak{g}, \mathfrak{h}$; $\operatorname{End}(V))$ (see, e.g., [3]).

Denote $\mathcal{D}:=\mathcal{D}(n, \delta)$ the $\operatorname{Vect}_{\mathrm{Pol}}(\mathbb{R})$-module of differential operators on $\mathcal{S}_{\delta}^{n}$. The infinitesimal deformations of the $\operatorname{Vect}_{\mathrm{Pol}}(\mathbb{R})$-module $\mathcal{S}_{\delta}^{n}$ are classified by the first differential cohomology space,

$$
\mathrm{H}_{\text {diff }}^{1}\left(\operatorname{Vect}_{\mathrm{Pol}}(\mathbb{R}) ; \mathcal{D}\right)=\oplus_{\lambda, k} \mathrm{H}_{\text {diff }}^{1}\left(\operatorname{Vect} \operatorname{Pol}_{(\mathbb{R})}\left(\mathcal{D}_{\lambda, \lambda+k}\right)\right.
$$

while the obstructions for integrability of infinitesimal deformations belong to the second differential cohomology space,

$$
\mathrm{H}_{\text {diff }}^{2}\left(\operatorname{Vect}_{\mathrm{Pol}}(\mathbb{R}) ; \mathcal{D}\right)=\oplus_{\lambda, k} \mathrm{H}_{\text {diff }}^{2}\left(\operatorname{Vect}_{\mathrm{Pol}}(\mathbb{R}) ; \mathcal{D}_{\lambda, \lambda+k}\right)
$$

where, hereafter, $\delta-\lambda$ and $k$ are integers satisfying $\delta-n \leq \lambda, \lambda+k \leq \delta$.

Here we study the $\mathfrak{s l}(2)$-trivial deformations, thus we consider the differential $\mathfrak{s l}(2)$-relative cohomology spaces. The first space

$$
\mathrm{H}_{\text {diff }}^{1}\left(\operatorname{Vect}_{\mathrm{Pol}}(\mathbb{R}), \mathfrak{s l}(2) ; \mathcal{D}\right)=\oplus_{\lambda, k} \mathrm{H}_{\text {diff }}^{1}\left(\operatorname{Vect}_{\mathrm{Pol}}(\mathbb{R}), \mathfrak{s l}(2) ; \mathcal{D}_{\lambda, \lambda+k}\right)
$$


was calculated by Bouarroudj and Ovsienko [4]; and the second space

$$
\mathrm{H}_{\text {diff }}^{2}\left(\operatorname{Vect}_{\mathrm{Pol}}(\mathbb{R}), \mathfrak{s l}(2) ; \mathcal{D}\right)=\oplus_{\lambda, k} \mathrm{H}_{\text {diff }}^{2}\left(\operatorname{Vect}_{\mathrm{Pol}}(\mathbb{R}), \mathfrak{s l}(2) ; \mathcal{D}_{\lambda, \lambda+k}\right)
$$

was calculated by Bouarroudj [3]. We give explicit expressions of some 2-cocycles that span the cohomology group $\mathrm{H}^{2}\left(\operatorname{Vect}_{\mathrm{P}}(\mathbb{R}), \mathfrak{s l}(2) ; \mathcal{D}_{\lambda, \lambda+k}\right)$.

This paper is organized as follows. In Section 2 we study some properties of the $\mathfrak{s l}(2)$-invariant differential operators. These properties are related to the $\mathfrak{s l}(2)$-relative cohomology. In Section 3 we study the first and the second $\mathfrak{s l}(2)$-relative cohomology spaces which are closely related to the deformation theory. Especially we explain some $\mathfrak{s l}(2)$-relative 2-cocycles which naturally appear as obstructions to integrate any $\mathfrak{s l}(2)$-trivial infinitesimal deformation to a formal one. In Section 4 we give an outline of the general deformation theory: definitions, equivalence, integrability conditions and miniversal deformations. In Section 5 we give the first main result of this paper: Theorem 2. That is, we explain all second-order integrability conditions for any infinitesimal $\mathfrak{s l}(2)$-trivial deformation of the $\operatorname{Vect}_{\text {Pol }}(\mathbb{R})$-module $\mathcal{S}_{\delta}^{n}$. In Section 6 we complete the list of integrability conditions by computing those of third and fourth-order. We prove that these conditions are necessary and sufficient to integrate any infinitesimal $\mathfrak{s l}(2)$-trivial deformation to a formal one. Moreover, we prove that any $\mathfrak{s l}(2)$-trivial deformation is, in fact, equivalent to a polynomial one of degree $\leq 2$ : Theorem 3. Finally, in Section 7 , we complete our study by giving a few examples of deformations.

\section{Invariant differential operators}

In this paper we study the $\mathfrak{s l}(2)$-trivial deformations of the space of symbols of differential

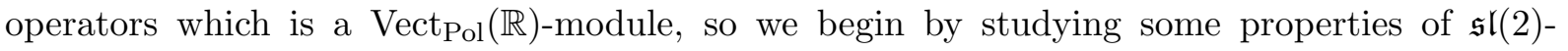
invariant bilinear differential operators.

Let us consider the space of bilinear differential operators $c: \mathcal{F}_{\lambda} \times \mathcal{F}_{\mu} \rightarrow \mathcal{F}_{\tau}$. The Lie algebra, $\operatorname{Vect}_{\mathrm{Pol}}(\mathbb{R})$, acts on this space by the Lie derivative:

$$
L_{X}(c)\left(f d x^{\lambda}, g d x^{\mu}\right)=L_{X}^{\tau}\left(c\left(f d x^{\lambda}, g d x^{\mu}\right)\right)-c\left(L_{X}^{\lambda}\left(f d x^{\lambda}\right), g d x^{\mu}\right)-c\left(f d x^{\lambda}, L_{X}^{\mu}\left(g d x^{\mu}\right)\right) .
$$

A bilinear differential operator $c: \mathcal{F}_{\tau} \times \mathcal{F}_{\lambda} \rightarrow \mathcal{F}_{\mu}$ is called $\mathfrak{s l}(2)$-invariant if, for all $X \in \mathfrak{s l}(2)$, we have $L_{X}(c)=0$, or equivalently

$$
L_{X}^{\mu}\left(c\left(f d x^{\tau}, g d x^{\lambda}\right)\right)=c\left(L_{X}^{\tau}\left(f d x^{\tau}\right), g d x^{\lambda}\right)+c\left(f d x^{\tau}, L_{X}^{\lambda}\left(g d x^{\lambda}\right)\right) .
$$

That is, the set of such $\mathfrak{s l}(2)$-invariant bilinear differential operators is the subspace on which the subalgebra $\mathfrak{s l}(2)$ acts trivially.

Now, let us consider a linear map $c: \operatorname{Vect}_{\mathrm{Pol}}(\mathbb{R}) \rightarrow \mathcal{D}_{\lambda, \mu}$, then we can see $c$ as a bilinear differential operator $c: \operatorname{Vect}_{\mathrm{Pol}}(\mathbb{R}) \times \mathcal{F}_{\lambda} \rightarrow \mathcal{F}_{\mu}\left(\right.$ or $c: \mathcal{F}_{-1} \times \mathcal{F}_{\lambda} \rightarrow \mathcal{F}_{\mu}$ since $\operatorname{Vect}_{\mathrm{Pol}}(\mathbb{R})$ is isomorphic to $\left.\mathcal{F}_{-1}\right)$ defined by $c\left(X, f d x^{\lambda}\right)=c(X)\left(f d x^{\lambda}\right)$. So, the $\mathfrak{s l}(2)$-invariance property (2) of $c$ reads, for all $X \in \mathfrak{s l}(2)$ and $Y \in \operatorname{Vect}_{\mathrm{Pol}}(\mathbb{R})$,

$$
L_{X}^{\mu} \circ c(Y)=c([X, Y])+c(Y) \circ L_{X}^{\lambda},
$$

or equivalently

$$
L_{X}^{\lambda, \mu}(c(Y))=c([X, Y]) .
$$

The $\mathfrak{s l}(2)$-invariant bilinear differential operators were calculated by Gordan. We recall here the results and we need to add some precision concerning the space of the $\mathfrak{s l}(2)$-invariant differential operators from $\operatorname{Vect}_{\mathrm{Pol}}(\mathbb{R})$ to $\mathcal{D}_{\lambda, \mu}$ vanishing on $\mathfrak{s l}(2)$. 
Proposition 1 ([11]). There exist $\mathfrak{s l}(2)$-invariant bilinear differential operators, called transvectants,

$$
J_{k}^{\tau, \lambda}: \quad \mathcal{F}_{\tau} \times \mathcal{F}_{\lambda} \rightarrow \mathcal{F}_{\tau+\lambda+k}, \quad\left(\varphi d x^{\tau}, \phi d x^{\lambda}\right) \mapsto J_{k}^{\tau, \lambda}(\varphi, \phi) d x^{\tau+\lambda+k}
$$

given by

$$
J_{k}^{\tau, \lambda}(\varphi, \phi)=\sum_{i+j=k} c_{i, j} \varphi^{(i)} \phi^{(j)}
$$

where $k \in \mathbb{N}$ and the coefficients $c_{i, j}$ are characterized as follows:

i) If neither $\tau$ nor $\lambda$ belong to the set $\left\{0,-\frac{1}{2},-1, \ldots,-\frac{k-1}{2}\right\}$ then

$$
c_{i, j}=(-1)^{j}\left(\begin{array}{c}
2 \tau+k \\
j
\end{array}\right)\left(\begin{array}{c}
2 \lambda+k \\
i
\end{array}\right)
$$

where $\left(\begin{array}{l}x \\ i\end{array}\right)$ is the standard binomial coefficient $\left(\begin{array}{l}x \\ i\end{array}\right)=\frac{x(x-1) \cdots(x-i+1)}{i !}$.

ii) If $\tau$ or $\lambda \in\left\{0,-\frac{1}{2},-1, \ldots,-\frac{k-1}{2}\right\}$, the coefficients $c_{i, j}$ satisfy the recurrence relation

$$
(i+1)(i+2 \tau) c_{i+1, j}+(j+1)(j+2 \lambda) c_{i, j+1}=0 .
$$

Moreover, the space of solutions of the system (3) is two-dimensional if $2 \lambda=-s$ and $2 \tau=-t$ with $t>k-s-2$, and one-dimensional otherwise.

iii) For $k \geq 3$, the space of $\mathfrak{s l}(2)$-invariant linear differential operators from $\operatorname{Vect}_{\mathrm{Pol}}(\mathbb{R})$ to $\mathcal{D}_{\lambda, \lambda+k-1}$ vanishing on $\mathfrak{s l}(2)$ is one-dimensional.

Proof. We need to prove only part iii), for the other statements see, for instance, [11] or [3]. First, we recall that $\operatorname{Vect}_{\mathrm{Pol}}(\mathbb{R})$ is isomorphic to $\mathcal{F}_{-1}$ as $\operatorname{Vect}_{\mathrm{Pol}}(\mathbb{R})$-module. So, according to the formulae (3), if $k \geq 3$, the space of $\mathfrak{s l}(2)$-invariant bilinear differential operator from $\operatorname{Vect}_{\mathrm{Pol}}(\mathbb{R}) \times \mathcal{F}_{\lambda}$ to $\mathcal{F}_{\lambda-1+k}$ is 2-dimensional if and only if $2 \lambda \in\{1-k, 2-k, 3-k\}$. Let us consider the transvectant $J_{k}^{-1, \lambda}$ defined by, for $\varphi \frac{d}{d x} \in \operatorname{Vect}_{\mathrm{Pol}}(\mathbb{R})$ and $\phi d x^{\lambda} \in \mathcal{F}_{\lambda}$,

$$
J_{k}^{-1, \lambda}(\varphi, \phi)=\sum_{i+j=k, i \geq 3} c_{i, j} \varphi^{(i)} \phi^{(j)}
$$

where the coefficients $c_{i, j}$ satisfy (3). If $2 \lambda \in\{1-k, 2-k, 3-k\}$ the space of $\mathfrak{s l}(2)$-invariant linear differential operator from $\operatorname{Vect}{ }_{\mathrm{Pol}}(\mathbb{R})$ to $\mathcal{D}_{\lambda, \lambda+k-1}$ is spanned by $J_{k}^{-1, \lambda}$ and $I_{k}^{-1, \lambda}$ where

$$
I_{k}^{-1, \lambda}(\varphi, \phi)= \begin{cases}\varphi \phi^{(k)} & \text { if } 2 \lambda=1-k \\ \varphi \phi^{(k)}+\frac{k}{2} \varphi^{\prime} \phi^{(k-1)} & \text { if } 2 \lambda=2-k \\ \varphi \phi^{(k)}+k \varphi^{\prime} \phi^{(k-1)}+\frac{k(k-1)}{2} \varphi^{\prime \prime} \phi^{(k-2)} & \text { if } 2 \lambda=3-k\end{cases}
$$

If $2 \lambda \notin\{1-k, 2-k, 3-k\}$ the corresponding space is one-dimensional and it is spanned by $J_{k}^{-1, \lambda}$. We see obviously that only the operators $J_{k}^{-1, \lambda}$ vanish on $\mathfrak{s l}(2)$. Part iii) of Proposition 1 is proved.

\section{Cohomology spaces}

Let $\mathfrak{g}$ be a Lie algebra acting on a space $V$ and let $\mathfrak{h}$ be a subalgebra of $\mathfrak{g}$. The space of $\mathfrak{h}$-relative $n$-cochains of $\mathfrak{g}$ with values in $V$ is the $\mathfrak{g}$-module

$$
C^{n}(\mathfrak{g}, \mathfrak{h} ; V):=\operatorname{Hom}_{\mathfrak{h}}\left(\wedge^{n}(\mathfrak{g} / \mathfrak{h}) ; V\right) .
$$


The coboundary operator $\partial^{n}: C^{n}(\mathfrak{g}, \mathfrak{h} ; V) \rightarrow C^{n+1}(\mathfrak{g}, \mathfrak{h} ; V)$ is a $\mathfrak{g}$-map satisfying $\partial^{n} \circ \partial^{n-1}=0$. The kernel of $\partial^{n}$, denoted $Z^{n}(\mathfrak{g}, \mathfrak{h} ; V)$, is the space of $\mathfrak{h}$-relative $n$-cocycles, among them, the elements in the range of $\partial^{n-1}$ are called $\mathfrak{h}$-relative $n$-coboundaries. We denote $B^{n}(\mathfrak{g}, \mathfrak{h} ; V)$ the space of $n$-coboundaries.

By definition, the $n^{\text {th }} \mathfrak{h}$-relative cohomolgy space is the quotient space

$$
H^{n}(\mathfrak{g}, \mathfrak{h} ; V)=Z^{n}(\mathfrak{g}, \mathfrak{h} ; V) / B^{n}(\mathfrak{g}, \mathfrak{h} ; V) .
$$

We will only need the formula of $\partial^{n}$ (which will be simply denoted $\partial$ ) in degrees 0,1 and 2: for $v \in C^{0}(\mathfrak{g}, \mathfrak{h} ; V)=V^{\mathfrak{h}}, \partial v(X):=X v$, for $b \in C^{1}(\mathfrak{g}, \mathfrak{h} ; V)$,

$$
\partial b(X, Y):=X b(Y)-Y b(X)-b([X, Y])
$$

and for $\Omega \in C^{2}(\mathfrak{g} ; \mathfrak{h}, V)$,

$$
\partial \Omega(X, Y, Z):=X \Omega(Y, Z)-\Omega([X, Y], Z)+\circlearrowleft(X, Y, Z),
$$

where $\circlearrowleft(X, Y, Z)$ denotes the summands obtained from the two written ones by the cyclic permutation of the symbols $X, Y, Z$.

In this paper, we are interested in the differential $\mathfrak{s l}(2)$-relative cohomology spaces

$$
\mathrm{H}_{\text {diff }}^{1}\left(\operatorname{Vect}_{\mathrm{Pol}}(\mathbb{R}), \mathfrak{s l}(2) ; \mathcal{D}_{\lambda, \lambda+k}\right) \quad \text { and } \quad \mathrm{H}_{\text {diff }}^{2}\left(\operatorname{Vect}_{\mathrm{Pol}}(\mathbb{R}), \mathfrak{s l}(2) ; \mathcal{D}_{\lambda, \lambda+k}\right) .
$$

\section{Proposition 2.}

i) Any 1-cocycle $c: \operatorname{Vect}_{\mathrm{Pol}}(\mathbb{R}) \rightarrow \mathcal{D}_{\lambda, \lambda+k}$ vanishing on $\mathfrak{s l}(2)$ coincides (up to a scalar factor) with the transvectant $J_{k+1}^{-1, \lambda}$ defined here and below by the formulae (4).

ii) Any 2-cocycle vanishing on $\mathfrak{s l}(2)$ is $\mathfrak{s l}(2)$-invariant.

iii) Let $\Omega \in Z^{2}\left(\operatorname{Vect}_{\mathrm{Pol}}(\mathbb{R}), \mathfrak{s l}(2) ; \mathcal{D}_{\lambda, \lambda+k}\right)$. If $\Omega$ is a $\mathfrak{s l}(2)$-relative 2-coboundary then (up to a scalar factor) we have $\Omega=\partial J_{k+1}^{-1, \lambda}$.

Proof. i) The 1-cocycle relation reads:

$$
c([X, Y])=L_{X}^{\mu} \circ c(Y)-c(Y) \circ L_{X}^{\lambda}-L_{Y}^{\mu} \circ c(X)+c(X) \circ L_{X}^{\lambda} .
$$

Consider $X \in \mathfrak{s l}(2)$. Since $c(X)=0$, one easily sees that

$$
L_{X}^{\mu} c(Y)=c([X, Y])+c(Y) \circ L_{X}^{\lambda} .
$$

The equation (6) expresses the $\mathfrak{s l}(2)$-invariance property of the bilinear map $c$. Thus, according to Proposition 1 , the map $c$ coincides with the transvectant $J_{k+1}^{-1, \lambda}$.

ii) Let $\Omega \in Z^{2}\left(\operatorname{Vect}_{\mathrm{Pol}}(\mathbb{R}) ; \mathcal{D}_{\lambda, \lambda+k}\right)$. Since $\Omega(X, Y)=0$ for all $X \in \mathfrak{s l}(2)$, we deduce from the 2-cocycle relation (5) that, for all $X \in \mathfrak{s l}(2)$ and $Y, Z \in \operatorname{Vect}_{\text {Pol }}(\mathbb{R})$, we have

$$
X \Omega(Y, Z)-\Omega([X, Y], Z)-\Omega(Y,[X, Z])=0 .
$$

This last relation is nothing but the $\mathfrak{s l}(2)$-invariance property of the bilinear map $\Omega$.

iii) Let $\Omega=\partial b$. For all $X, Y \in \operatorname{Vect}_{\mathrm{Pol}}(\mathbb{R})$ we have

$$
\partial b(X, Y):=L_{X}^{\lambda, \lambda+k} b(Y)-L_{Y}^{\lambda, \lambda+k} b(X)-b([X, Y]) .
$$

Since $\partial b(X, Y)=b(X)=0$ for all $X \in \mathfrak{s l}(2)$ we deduce that $b$ is $\mathfrak{s l}(2)$-invariant:

$$
L_{X}^{\lambda, \lambda+k} b(Y)=b([X, Y]) .
$$

According to Proposition 1, the space of $\mathfrak{s l}(2)$-invariant linear differential operator from $\operatorname{Vect}_{\text {Pol }}(\mathbb{R})$ to $\mathcal{D}_{\lambda, \lambda+k}$ vanishing on $\mathfrak{s l}(2)$ is one dimensional and it is spanned by $J_{k+1}^{-1, \lambda}$. Thus, up to a scalar factor, $b=J_{k+1}^{-1, \lambda}$. Proposition 2 is proved. 
The $\mathfrak{s l}(2)$-trivial deformations are closely related to the $\mathfrak{s l}(2)$-relative cohomology spaces $\mathrm{H}_{\text {diff }}^{1}\left(\operatorname{Vect}_{\text {Pol }}(\mathbb{R}), \mathfrak{s l}(2) ; \mathcal{D}_{\lambda, \lambda+k}\right)$ and $\mathrm{H}_{\text {diff }}^{2}\left(\operatorname{Vect}_{\text {Pol }}(\mathbb{R}), \mathfrak{s l}(2) ; \mathcal{D}_{\lambda, \lambda+k}\right)$. Therefore, we will describe briefly these two spaces.

\subsection{The first cohomology space}

Note that, by Proposition 2, we can describe the space $\mathrm{H}_{\text {diff }}^{1}\left(\operatorname{Vect}_{\text {Pol }}(\mathbb{R}), \mathfrak{s l}(2) ; \mathcal{D}_{\lambda, \lambda+k}\right)$. This space is, in fact, one-dimensional if and only if the corresponding transvectant $J_{k+1}^{-1, \lambda}$ is a nontrivial $\mathfrak{s l}(2)$-relative 1-cocycle, otherwise it is trivial. However, this space was computed by Bouarroudj and Ovsienko, the result is as follows:

Theorem 1 ([4]). $\operatorname{dimH}_{\text {diff }}^{1}\left(\operatorname{Vect}_{\mathrm{Pol}}(\mathbb{R}), \mathfrak{s l}(2) ; \mathcal{D}_{\lambda, \mu}\right)=1$ if

$$
\begin{array}{llll}
\mu-\lambda=2 & \text { and } & \lambda \neq-\frac{1}{2}, \\
\mu-\lambda=3 & \text { and } & \lambda \neq-1, \\
\mu-\lambda=4 & \text { and } & \lambda \neq-\frac{3}{2}, \\
\mu-\lambda=5 & \text { and } & \lambda=0,-4, \\
\mu-\lambda=6 & \text { and } & \lambda=-\frac{5 \pm \sqrt{19}}{2} .
\end{array}
$$

Otherwise, $\mathrm{H}_{\text {diff }}^{1}\left(\operatorname{Vect}_{\mathrm{Pol}}(\mathbb{R}), \mathfrak{s l}(2) ; \mathcal{D}_{\lambda, \mu}\right)=0$.

These spaces $\mathrm{H}_{\text {diff }}^{1}\left(\operatorname{Vect}_{\mathrm{Pol}}(\mathbb{R}), \mathfrak{s l}(2) ; \mathcal{D}_{\lambda, \lambda+k}\right)$ are generated by the cohomology classes of the $\mathfrak{s l}(2)$-relative 1-cocycles, $C_{\lambda, \lambda+k}: \operatorname{Vect} \operatorname{Pol}_{(\mathbb{R})} \rightarrow \mathcal{D}_{\lambda, \lambda+k}$ that are collected in the following table.

Table 1.

\begin{tabular}{|l|}
\hline$C_{\lambda, \lambda+2}(X, f)=X^{(3)} f, \quad \lambda \neq-\frac{1}{2}$ \\
\hline$C_{\lambda, \lambda+3}(X, f)=X^{(3)} f^{\prime}-\frac{\lambda}{2} X^{(4)} f, \quad \lambda \neq-1$ \\
\hline$C_{\lambda, \lambda+4}(X, f)=X^{(3)} f^{\prime \prime}-\frac{2 \lambda+1}{2} X^{(4)} f^{\prime}+\frac{\lambda(2 \lambda+1)}{10} X^{(5)} f, \quad \lambda \neq-\frac{3}{2}$ \\
\hline$C_{0,5}(X, f)=-3 X^{(5)} f^{\prime}+15 X^{(4)} f^{\prime \prime}-10 X^{(3)} f^{(3)}$ \\
\hline$C_{-4,1}(X, f)=28 X^{(6)} f+63 X^{(5)} f^{\prime}+45 X^{(4)} f^{\prime \prime}+10 X^{(3)} f^{(3)}$ \\
\hline$C_{a_{i}, a_{i}+6}(X, f)=\alpha_{i} X^{(7)} f-14 \beta_{i} X^{(6)} f^{\prime}-126 \gamma_{i} X^{(5)} f^{\prime \prime}-210 \tau_{i} X^{(4)} f^{(3)}+210 X^{(3)} f^{4}$ \\
\hline
\end{tabular}

where

$$
\begin{array}{lllll}
a_{1}=-\frac{5+\sqrt{19}}{2}, & \alpha_{1}=-\frac{22+5 \sqrt{19}}{4}, & \beta_{1}=\frac{31+7 \sqrt{19}}{2}, & \gamma_{1}=\frac{25+7 \sqrt{19}}{2}, & \tau_{1}=-2+\sqrt{19}, \\
a_{2}=-\frac{5-\sqrt{19}}{2}, & \alpha_{2}=-\frac{22-5 \sqrt{19}}{4}, & \beta_{2}=\frac{31-7 \sqrt{19}}{2}, & \gamma_{2}=\frac{25-7 \sqrt{19}}{2}, & \tau_{2}=-2-\sqrt{19} .
\end{array}
$$

For $X \frac{d}{d x} \in \operatorname{Vect}_{\mathrm{Pol}}(\mathbb{R})$ and $f d x^{\lambda} \in \mathcal{F}_{\lambda}$, we write

$$
C_{\lambda, \lambda+k}\left(X \frac{d}{d x}\right)\left(f d x^{\lambda}\right)=C_{\lambda, \lambda+k}(X, f) d x^{\lambda+k} .
$$

The maps $C_{\lambda, \lambda+j}(X)$ are naturally extended to $\mathcal{S}_{\delta}^{n}=\bigoplus_{j=0}^{n} \mathcal{F}_{\delta-j}$.

\subsection{The second cohomology space}

Let $\mathfrak{g}$ be a Lie algebra, $\mathfrak{h}$ a subalgebra of $\mathfrak{g}$ and $V$ a $\mathfrak{g}$-module, the cup-product is defined, for arbitrary linear maps $c_{1}, c_{2}: \mathfrak{g} \rightarrow \operatorname{End}(V)$, by

$$
\llbracket c_{1}, c_{2} \rrbracket: \quad \mathfrak{g} \otimes \mathfrak{g} \rightarrow \operatorname{End}(V), \quad \llbracket c_{1}, c_{2} \rrbracket(x, y)=\left[c_{1}(x), c_{2}(y)\right]+\left[c_{2}(x), c_{1}(y)\right] .
$$


It is easy to check that for any two $\mathfrak{h}$-relative 1-cocycles $c_{1}$ and $c_{2} \in Z^{1}(\mathfrak{g}, \mathfrak{h} ; \operatorname{End}(V))$, the bilinear map $\llbracket c_{1}, c_{2} \rrbracket$ is a $\mathfrak{h}$-relative 2-cocycle. Moreover, if one of the cocycles $c_{1}$ or $c_{2}$ is a $\mathfrak{h}$-relative 1-coboundary, then $\llbracket c_{1}, c_{2} \rrbracket$ is a $\mathfrak{h}$-relative 2-coboundary. Therefore, we naturally deduce that the operation (7) defines a bilinear map

$$
\mathrm{H}^{1}(\mathfrak{g}, \mathfrak{h} ; \operatorname{End}(V)) \otimes \mathrm{H}^{1}(\mathfrak{g}, \mathfrak{h} ; \operatorname{End}(V)) \rightarrow \mathrm{H}^{2}(\mathfrak{g}, \mathfrak{h} ; \operatorname{End}(V)) .
$$

Thus, by computing the cup-products of the 1-cocycles $C_{\lambda, \lambda+k}$ generating the spaces

$$
\mathrm{H}_{\text {diff }}^{1}\left(\operatorname{Vect}_{\mathrm{Pol}}(\mathbb{R}), \mathfrak{s l}(2) ; \mathcal{D}_{\lambda, \lambda+k}\right),
$$

we can exhibit explicit expressions of some $\mathfrak{s l}(2)$-relative 2-cocycles

$$
\Omega_{\lambda, \lambda+k}: \operatorname{Vect}_{\mathrm{Pol}}(\mathbb{R}) \rightarrow \mathcal{D}_{\lambda, \lambda+k} .
$$

For $X \frac{d}{d x}, Y \frac{d}{d x} \in \operatorname{Vect}_{\mathrm{Pol}}(\mathbb{R})$ and $f d x^{\lambda} \in \mathcal{F}_{\lambda}$, we write

$$
\Omega_{\lambda, \lambda+k}\left(X \frac{d}{d x}, Y \frac{d}{d x}\right)\left(f d x^{\lambda}\right)=\Omega_{\lambda, \lambda+k}(X, Y, f) d x^{\lambda+k} .
$$

\section{Proposition 3.}

i) The map $\Omega_{\lambda, \lambda+5}$ is defined by

$$
(\lambda+4) \Omega_{\lambda, \lambda+5}=2 \llbracket C_{\lambda+2, \lambda+5}, C_{\lambda, \lambda+2} \rrbracket
$$

is a nontrivial $\mathfrak{s l}(2)$-relative 2 -cocycle if and only if $\lambda \in\{0,-2,-4\}$. Moreover

$$
-2 \llbracket C_{\lambda+3, \lambda+5}, C_{\lambda, \lambda+3} \rrbracket=\lambda \Omega_{\lambda, \lambda+5} .
$$

ii) The map $\Omega_{\lambda, \lambda+6}$ is defined by

$$
(2 \lambda+9) \Omega_{\lambda, \lambda+6}=-2 \llbracket C_{\lambda+2, \lambda+6}, C_{\lambda, \lambda+2} \rrbracket
$$

is a nontrivial $\mathfrak{s l}(2)$-relative 2 -cocycle if and only if $\lambda \in\left\{-\frac{5}{2},-\frac{22-5 \sqrt{19}}{4},-\frac{22+5 \sqrt{19}}{4}\right\}$. Moreover,

$$
5(2 \lambda+1) \Omega_{\lambda, \lambda+6}=-2(2 \lambda+1) \llbracket C_{\lambda+3, \lambda+6}, C_{\lambda, \lambda+3} \rrbracket=10 \llbracket C_{\lambda+4, \lambda+6}, C_{\lambda, \lambda+4} \rrbracket .
$$

Proof. By a straightforward computation we get

$$
\begin{aligned}
& \Omega_{\lambda, \lambda+5}(X, Y, f)=\left(X^{(4)} Y^{(3)}-X^{(3)} Y^{(4)}\right) f \\
& \Omega_{\lambda, \lambda+6}(X, Y, f)=\left(X^{(3)} Y^{(4)}-X^{(4)} Y^{(3)}\right) f^{\prime}-\frac{\lambda}{5}\left(X^{(3)} Y^{(5)}-X^{(5)} Y^{(3)}\right) f .
\end{aligned}
$$

Moreover, we show also by a direct computation that

$$
3 \partial J_{6}^{-1, \lambda}=-\lambda\left(\lambda^{2}+6 \lambda+8\right) \Omega_{\lambda, \lambda+5}, \quad \text { and } \quad 3 \partial J_{7}^{-1, \lambda}=\left(4 \lambda^{3}+30 \lambda^{2}+56 \lambda+15\right) \Omega_{\lambda, \lambda+6}
$$

where

$$
J_{6}^{-1, \lambda}(X)(f)=3 X^{(3)} f^{(3)}-\frac{9}{2}(\lambda+1) X^{(4)} f^{\prime \prime}+\frac{9}{10}(\lambda+1)(2 \lambda+1) X^{(5)} f^{\prime}-\lambda \frac{2 \lambda^{2}+3 \lambda+1}{10} X^{(6)} f
$$

and

$$
\begin{aligned}
J_{7}^{-1, \lambda}(X)(f)= & X^{(3)} f^{(4)}-(2 \lambda+3) X^{(4)} f^{(3)}+\frac{6 \lambda^{2}+15 \lambda+9}{5} X^{(5)} f^{\prime \prime} \\
& -\frac{4 \lambda^{3}+12 \lambda^{2}+11 \lambda+3}{15} X^{(6)} f^{\prime}+\frac{\lambda\left(4 \lambda^{3}+12 \lambda^{2}+11 \lambda+3\right)}{210} X^{(7)} f .
\end{aligned}
$$

Thus, we conclude by using Proposition 2. 
Proposition 4. The cup products $\llbracket C_{\lambda+3, \lambda+7}, C_{\lambda, \lambda+3} \rrbracket$ and $\llbracket C_{\lambda+4, \lambda+7}, C_{\lambda, \lambda+4} \rrbracket$ are generically nontrivial $\mathfrak{s l}(2)$-relative 2 -cocycles and they are cohomologous.

Proof. The transvectant $J_{8}^{-1, \lambda}$ is given by, for $X \frac{d}{d x} \in \operatorname{Vect}_{\mathrm{Pol}}(\mathbb{R})$ and $f d x^{\lambda} \in \mathcal{F}_{\lambda}$,

$$
\begin{aligned}
J_{8}^{-1, \lambda}(X)(f)= & X^{(3)} f^{(5)}-\frac{5}{2}(\lambda+2) X^{(4)} f^{(4)}+(\lambda+2)(2 \lambda+3) X^{(5)} f^{(3)} \\
& -\frac{1}{3}(\lambda+1)(\lambda+2)(2 \lambda+3) X^{(6)} f^{\prime \prime}+\frac{1}{42}(\lambda+1)(\lambda+2)(2 \lambda+3)(2 \lambda+1) X^{(7)} f^{\prime} \\
& -\frac{1}{840} \lambda(\lambda+1)(\lambda+2)(2 \lambda+3)(2 \lambda+1) X^{(8)} f .
\end{aligned}
$$

Therefore, by a direct computation, we show that

$$
\begin{aligned}
\partial J_{8}^{-1, \lambda} & (X, Y)(f)=\frac{1}{30} \lambda((\lambda+1)(\lambda+2)(2 \lambda+3)(2 \lambda+11)+30) X^{(3)} Y^{(6)} f \\
& -\lambda(\lambda+2)\left(-\frac{1}{60}(\lambda+1)(2 \lambda+3)(2 \lambda+1)+2 \lambda+\frac{11}{2}\right) X^{(4)} Y^{(5)} f \\
& -\left((\lambda+2)(2 \lambda+3)\left(\frac{1}{3}(\lambda+1)(2 \lambda+1)+3 \lambda+1\right)-5 \lambda-1\right) X^{(3)} Y^{(5)} f^{\prime} \\
& +5\left((\lambda+2)\left[\frac{1}{3}(\lambda+1)(2 \lambda+3)+3 \lambda+2\right]+2 \lambda+1\right) X^{(3)} Y^{(4)} f^{\prime \prime}-(X \leftrightarrow Y) .
\end{aligned}
$$

Let us define $\Omega_{\lambda, \lambda+7}$ and $\widetilde{\Omega}_{\lambda, \lambda+7}$ by

$$
\Omega_{\lambda, \lambda+7}=\llbracket C_{\lambda+3, \lambda+7}, C_{\lambda, \lambda+3} \rrbracket \quad \text { and } \quad \widetilde{\Omega}_{\lambda, \lambda+7}=\llbracket C_{\lambda+4, \lambda+7}, C_{\lambda, \lambda+4} \rrbracket .
$$

Thus,

$$
\begin{aligned}
\Omega_{\lambda, \lambda+7}(X, Y, f)= & \left(\frac{-\lambda(2 \lambda+7)(\lambda+8)}{20} X^{(5)} Y^{(4)}-\frac{\lambda}{2} X^{(3)} Y^{(6)}\right) f+\frac{2 \lambda^{2}+23 \lambda+11}{10} X^{(5)} Y^{(3)} f^{\prime} \\
& +\frac{\lambda+11}{2} X^{(3)} Y^{(4)} f^{\prime \prime}-(X \leftrightarrow Y), \\
\widetilde{\Omega}_{\lambda, \lambda+7}(X, Y, f)= & \left(\frac{\lambda(2 \lambda+1)}{10} X^{(3)} Y^{(6)}-\frac{\lambda(\lambda+4)(2 \lambda+1)}{20} X^{(4)} Y^{(5)}\right) f+\frac{(\lambda-5)(2 \lambda+1)}{10} X^{(3)} Y^{(5)} f^{\prime} \\
& +\frac{5-\lambda}{2} X^{(3)} Y^{(4)} f^{\prime \prime}-(X \leftrightarrow Y) .
\end{aligned}
$$

We exhibit some reals $a, b$ and $c$ such that:

$$
a \Omega_{\lambda, \lambda+7}+b \widetilde{\Omega}_{\lambda, \lambda+7}=c \partial J_{8}^{-1, \lambda} .
$$

For $\lambda=-3$, we get $\Omega_{\lambda, \lambda+7}=\widetilde{\Omega}_{\lambda, \lambda+7}$ and $\partial J_{8}^{-1, \lambda}=0$. For $\lambda=-6$, we get $a=1$ and $70 c=5+11 b$. For $\lambda \in\left\{-5,-\frac{3}{2},-\frac{1}{2}\right\}$, we get

$$
a=0, \quad b=1, \quad \text { and } \quad 210 c=-8 \lambda^{3}-60 \lambda^{2}-70 \lambda+45 .
$$

For $\lambda \notin\left\{-6,-5,-3,-\frac{3}{2}, \frac{1}{2}\right\}$, we get

$$
a=1, \quad b=\frac{4 \lambda^{3}+48 \lambda^{2}+161 \lambda+117}{4 \lambda^{3}+24 \lambda^{2}+17 \lambda-15} \quad \text { and } \quad c=\frac{b\left(4 \lambda^{3}+24 \lambda^{2}+3 \lambda-15\right)-4 \lambda^{3}-48 \lambda^{2}-147 \lambda-33}{70(\lambda+3)} .
$$

Now, it is easy to show that, if $4 \lambda^{3}+48 \lambda^{2}+161 \lambda+117 \neq 0$ the 2 -cocycle $\Omega_{\lambda, \lambda+7}$ is nontrivial: $\Omega_{\lambda, \lambda+7} \neq \partial J_{8}^{-1, \lambda}$. Similarly, if $\lambda \notin\left\{-5,-\frac{3}{2}, \frac{1}{2}\right\}$, we show that $\widetilde{\Omega}_{\lambda, \lambda+7}$ is nontrivial.

Proposition 5. The cup product $\llbracket C_{\lambda, \lambda+4}, C_{\lambda+4, \lambda+8} \rrbracket$ is a nontrivial $\mathfrak{s l}(2)$-relative 2-cocycle.

Proof. Let $\Omega_{\lambda, \lambda+8}=\llbracket C_{\lambda, \lambda+4}, C_{\lambda+4, \lambda+8} \rrbracket$. By a straightforward computation we show that

$$
\begin{aligned}
\Omega_{\lambda, \lambda+8}(X, Y, f)= & -\left(\frac{\lambda(2 \lambda+1)(2 \lambda+9)}{20} X^{(4)} Y^{(6)}-\frac{\lambda(2 \lambda+1)}{10} X^{(3)} Y^{(7)}\right) f \\
& -\left(\frac{9(2 \lambda+1)(2 \lambda+9)}{20} X^{(5)} Y^{(4)}-\frac{(2 \lambda+1)(2 \lambda-5)}{10} X^{(3)} Y^{(6)}\right) f^{\prime} \\
& +\frac{18(1+\lambda)}{5} X^{(5)} Y^{(3)} f^{\prime \prime}-6 X^{(4)} Y^{(3)} f^{(3)}-(X \leftrightarrow Y) .
\end{aligned}
$$

As before, we show that this 2-cocycle $\Omega_{\lambda, \lambda+8}$ is nontrivial: $\Omega_{\lambda, \lambda+8} \neq \partial J_{9}^{-1, \lambda}$. 
Now, we collect in the following proposition some $\mathfrak{s l}(2)$-relative nontrivial 2 -cocycles $\Omega_{\lambda, \lambda+k}$ for $k=9,10$ and for singular values of $\lambda$.

Proposition 6. The following cup-products are $\mathfrak{s l}(2)$-relative nontrivial 2-cocycles

$$
\begin{array}{ll}
\Omega_{0,9}=\llbracket C_{0,5}, C_{5,9} \rrbracket, & \Omega_{-4,5}=\llbracket C_{-4,0}, C_{0,5} \rrbracket, \\
\Omega_{-8,1}=\llbracket C_{-8,-4}, C_{-4,1} \rrbracket, & \Omega_{a_{i}, a_{i}+9}=\llbracket C_{a_{i}, a_{i}+6}, C_{a_{i}+6, a_{i}+9} \rrbracket, \\
\Omega_{a_{i}-3, a_{i}+6}=\llbracket C_{a_{i}-3, a_{i}}, C_{a_{i}, a_{i}+6} \rrbracket, & \Omega_{a_{i}, a_{i}+10}=\llbracket C_{a_{i}, a_{i}+6}, C_{a_{i}+6, a_{i}+10} \rrbracket, \\
\Omega_{a_{i}-4, a_{i}+6}=\llbracket C_{a_{i}-4, a_{i}}, C_{a_{i}, a_{i}+6} \rrbracket . &
\end{array}
$$

\section{The general framework}

In this section we define deformations of Lie algebra homomorphisms and introduce the notion of miniversal deformations over complete local algebras. Deformation theory of Lie algebra homomorphisms was first considered with only one-parameter deformation $[7,12,15]$. Recently, deformations of Lie (super)algebras with multi-parameters were intensively studied (see, e.g., [1, $2,13,14])$. Here we give an outline of this theory.

\subsection{Infinitesimal deformations}

Let $\rho_{0}: \mathfrak{g} \rightarrow \operatorname{End}(V)$ be an action of a Lie algebra $\mathfrak{g}$ on a vector space $V$ and let $\mathfrak{h}$ be a subagebra of $\mathfrak{g}$. When studying $\mathfrak{h}$-trivial deformations of the $\mathfrak{g}$-action $\rho_{0}$, one usually starts with infinitesimal deformations

$$
\rho=\rho_{0}+t C,
$$

where $C: \mathfrak{g} \rightarrow \operatorname{End}(V)$ is a linear map vanishing on $\mathfrak{h}$ and $t$ is a formal parameter. The homomorphism condition

$$
[\rho(x), \rho(y)]=\rho([x, y])
$$

where $x, y \in \mathfrak{g}$, is satisfied in order 1 in $t$ if and only if $C$ is a $\mathfrak{h}$-relative 1 -cocycle. That is, the map $C$ satisfies

$$
\left[\rho_{0}(x), C(y)\right]-\left[\rho_{0}(y), C(x)\right]-C([x, y])=0 .
$$

Moreover, two $\mathfrak{h}$-trivial infinitesimal deformations $\rho=\rho_{0}+t C_{1}$, and $\rho=\rho_{0}+t C_{2}$, are equivalents if and only if $C_{1}-C_{2}$ is a $\mathfrak{h}$-relative coboundary:

$$
\left(C_{1}-C_{2}\right)(x)=\left[\rho_{0}(x), A\right]:=\partial A(x)
$$

where $A \in \operatorname{End}(V)^{\mathfrak{h}}$ and $\partial$ stands for differential of cochains on $\mathfrak{g}$ with values in $\operatorname{End}(V)$. So, the space $\mathrm{H}^{1}(\mathfrak{g}, \mathfrak{h}$; End $(V))$ determines and classifies the $\mathfrak{h}$-trivial infinitesimal deformations up to equivalence. (see, e.g., $[8,12]$ ). If $\mathrm{H}^{1}(\mathfrak{g}, \mathfrak{h}$; $\operatorname{End}(V))$ is multi-dimensional, it is natural to consider multi-parameter $\mathfrak{h}$-trivial deformations. More precisely, if $\operatorname{dim}^{1}(\mathfrak{g}, \mathfrak{h} ; \operatorname{End}(V))=m$, then choose $\mathfrak{h}$-relative 1-cocycles $C_{1}, \ldots, C_{m}$ representing a basis of $\mathrm{H}^{1}(\mathfrak{g}, \mathfrak{h} ; \operatorname{End}(V))$ and consider the $\mathfrak{h}$ trivial infinitesimal deformation

$$
\rho=\rho_{0}+\sum_{i=1}^{m} t_{i} C_{i},
$$

with independent parameters $t_{1}, \ldots, t_{m}$. 
In our study, we are interested in the infinitesimal $\mathfrak{s l}(2)$-trivial deformation of the $\operatorname{Vect}_{\mathrm{Pol}}(\mathbb{R})$ action on $\mathcal{S}_{\delta}^{n}=\bigoplus_{j=0}^{n} \mathcal{F}_{\delta-j}$, the space of symbols of differential operators, where $n \in \mathbb{N}$ and $\delta \in \mathbb{R}$. Thus, we consider the $\mathfrak{s l}(2)$-relative cohomology space $\mathrm{H}_{\text {diff }}^{1}(\operatorname{Vect} \operatorname{Pol}(\mathbb{R}), \mathfrak{s l}(2) ; \mathcal{D})$. Any infinitesimal $\mathfrak{s l}(2)$-trivial deformation is then of the form

$$
\mathcal{L}_{X}=L_{X}+\mathcal{L}_{X}^{(1)}
$$

where $L_{X}$ is the Lie derivative of $\mathcal{S}_{\delta}^{n}$ along the vector field $X \frac{d}{d x}$ defined by (1), and

$$
\mathcal{L}_{X}^{(1)}=\sum_{\lambda} \sum_{j=2}^{6} t_{\lambda, \lambda+j} C_{\lambda, \lambda+j}(X)
$$

and where $t_{\lambda, \lambda+j}$ are independent parameters, $\delta-\lambda \in \mathbb{N}, \delta-n \leq \lambda, \lambda+j \leq \delta$ and the $\mathfrak{s l}(2)$-relative 1-cocycles $C_{\lambda, \lambda+j}$ are defined in Table 1 .

Note that for $(j, \lambda)=\left(2,-\frac{1}{2}\right),(3,-1),\left(4,-\frac{3}{2}\right)$, or $j=5$ and $\lambda \notin\{0,-4\}$ or $j=6$ and $\lambda \neq-\frac{5 \pm \sqrt{19}}{2}$ we have $C_{\lambda, \lambda+j}=0$, then there are no corresponding parameters $t_{\lambda, \lambda+j}$.

\subsection{Integrability conditions}

Consider the problem of integrability of infinitesimal deformations. Starting with the infinitesimal deformation (9), we look for a formal series

$$
\mathcal{L}_{X}=L_{X}+\mathcal{L}_{X}^{(1)}+\mathcal{L}_{X}^{(2)}+\mathcal{L}_{X}^{(3)}+\cdots
$$

where $\mathcal{L}_{X}^{(k)}$ is an homogenous polynomial of degree $k$ in the parameters $\left(t_{\lambda, \lambda+j}\right)$ and with coefficients in $\mathcal{D}$ such that $\mathcal{L}_{X}^{(k)}=0$ if $X \frac{d}{d x} \in \mathfrak{s l}(2)$. This formal series (11) must satisfy the homomorphism condition in any order in the parameters $\left(t_{\lambda, \lambda+j}\right)$

$$
\left[\mathcal{L}_{X}, \mathcal{L}_{Y}\right]=\mathcal{L}_{[X, Y]}
$$

The homomorphism condition (12) gives the following (Maurer-Cartan) equations

$$
\partial \mathcal{L}^{(k)}=-\frac{1}{2} \sum_{i+j=k} \llbracket \mathcal{L}^{(i)}, \mathcal{L}^{(j)} \rrbracket
$$

However, quite often the above problem has no solution. Note here that the right side of (13) must be a coboundary of a 1-cochain vanishing on $\mathfrak{s l}(2)$, so, the obstructions for integrability of infinitesimal deformations belong to the second $\mathfrak{s l}(2)$-relative cohomology space $\mathrm{H}_{\text {diff }}^{2}(\operatorname{Vect} \operatorname{Pol}(\mathbb{R}), \mathfrak{s l}(2) ; \mathcal{D})$.

Following [7] and [2], we will impose extra algebraic relations on the parameters $\left(t_{\lambda, \lambda+j}\right)$. Let $\mathcal{R}$ be an ideal in $\mathbb{C}\left[\left[t_{\lambda, \lambda+j}\right]\right]$ generated by some set of relations, the quotient

$$
\mathcal{A}=\mathbb{C}\left[\left[t_{\lambda, \lambda+j}\right]\right] / \mathcal{R}
$$

is a complete local algebra with unity, and one can speak about deformations with base $\mathcal{A}$, see [7] for details.

Given an infinitesimal deformation (9), one can always consider it as a deformation with base (14), where $\mathcal{R}$ is the ideal generated by all the quadratic monomials. Our aim is to find $\mathcal{A}$ which is big as possible, or, equivalently, we look for relations on the parameters $\left(t_{\lambda, \lambda+j}\right)$ which are necessary and sufficient for integrability (cf. [1, 2]). 


\subsection{Equivalence and the miniversal deformation}

The notion of equivalence of deformations over complete local algebras has been considered in [6].

Definition 1. Two deformations, $\rho$ and $\rho^{\prime}$ with the same base $\mathcal{A}$ are called equivalent if there exists an inner automorphism $\Psi$ of the associative algebra $\operatorname{End}(V) \otimes \mathcal{A}$ such that

$$
\Psi \circ \rho=\rho^{\prime} \text { and } \Psi(\mathbb{I})=\mathbb{I},
$$

where $\mathbb{I}$ is the unity of the algebra $\operatorname{End}(\mathrm{V}) \otimes \mathcal{A}$.

The following notion of miniversal deformation is fundamental. It assigns to a $\mathfrak{g}$-module $V$ a canonical commutative associative algebra $\mathcal{A}$ and a canonical deformation with base $\mathcal{A}$.

Definition 2. A deformation $\rho$ with base $\mathcal{A}$ is called miniversal, if

(i) for any other deformation, $\rho^{\prime}$ with base (local) $\mathcal{A}^{\prime}$, there exists a homomorphism $\psi: \mathcal{A}^{\prime} \rightarrow \mathcal{A}$ satisfying $\psi(1)=1$, such that

$$
\rho=(\mathbb{I} d \otimes \psi) \circ \rho^{\prime} .
$$

(ii) in the notations of (i), if $\mathcal{A}$ is infinitesimal then $\psi$ is unique.

If $\rho$ satisfies only the condition (i), then it is called versal.

We refer to [7] for a construction of miniversal deformations of Lie algebras and to [2] for miniversal deformations of $\mathfrak{g}$-modules.

\section{Second-order integrability conditions}

In this section we obtain the integrability conditions for the infinitesimal deformation (9). Assume that the infinitesimal deformation (9) can be integrated to a formal deformation

$$
\mathcal{L}_{X}=L_{X}+\mathcal{L}_{X}^{(1)}+\mathcal{L}_{X}^{(2)}+\mathcal{L}_{X}^{(3)}+\cdots,
$$

where $\mathcal{L}_{X}^{(1)}$ is given by $(10)$ and $\mathcal{L}_{X}^{(2)}$ is a quadratic polynomial in $t$ whose coefficients are elements of $\mathcal{D}$ vanishing on $\mathfrak{s l}(2)$. We compute the conditions for the second-order terms $\mathcal{L}^{(2)}$. The homomorphism condition

$$
\left[\mathcal{L}_{X}, \mathcal{L}_{Y}\right]=\mathcal{L}_{[X, Y]}
$$

gives for the second-order terms the following (Maurer-Cartan) equation

$$
\partial \mathcal{L}^{(2)}=-\frac{1}{2} \llbracket \mathcal{L}^{(1)}, \mathcal{L}^{(1)} \rrbracket
$$

The right hand side of (15) is a cup-product of $\mathfrak{s l}(2)$-relative 1-cocycles, so it is automatically a $\mathfrak{s l}(2)$-relative 2-cocycle. More precisely, the equation (15) can be expressed as follows

$$
\partial \mathcal{L}^{(2)}=-\frac{1}{2} \llbracket \sum_{\lambda} \sum_{j=2}^{6} t_{\lambda, \lambda+j} C_{\lambda, \lambda+j}, \sum_{\lambda} \sum_{j=2}^{6} t_{\lambda, \lambda+j} C_{\lambda, \lambda+j} \rrbracket,
$$


therefore, let us consider the $\mathfrak{s l}(2)$-relative 2-cocycles $B_{\lambda, \lambda+k} \in Z_{\text {diff }}^{2}\left(\operatorname{Vect}_{\text {Pol }}(\mathbb{R}), \mathfrak{s l}(2), \mathcal{D}_{\lambda, \lambda+k}\right)$, for $k=4, \ldots, 10$, defined by

$$
B_{\lambda, \lambda+k}=-\sum_{j=2}^{k} t_{\lambda+j, \lambda+k} t_{\lambda, \lambda+j} \llbracket C_{\lambda+j, \lambda+k}, C_{\lambda, \lambda+j} \rrbracket .
$$

It is easy to see that $B_{\lambda, \lambda+4}=0$. The second order integrability conditions are determined by the fact that any map 2-cocycles $B_{\lambda, \lambda+k}$, for $k=5, \ldots, 10$, must be a $\mathfrak{s l}(2)$-relative 2coboundary. More precisely, $B_{\lambda, \lambda+k}$ must coincide, up to a scalar factor, with $\partial J_{k+1}^{-1, \lambda}$. We split these conditions into two family which we explain in the two following propositions. Let us first consider the following functions in $t$ where $t$ is the family of parameters $\left(t_{\lambda, \lambda+j}\right)$

$$
\begin{aligned}
& \omega_{\lambda, \lambda+5}(t)=-\frac{\lambda+4}{2} t_{\lambda, \lambda+2} t_{\lambda+2, \lambda+5}+\frac{\lambda}{2} t_{\lambda, \lambda+3} t_{\lambda+3, \lambda+5}, \\
& \omega_{\lambda, \lambda+6}(t)=\frac{2 \lambda+9}{2} t_{\lambda, \lambda+2} t_{\lambda+2, \lambda+6}+\frac{5}{2} t_{\lambda, \lambda+3} t_{\lambda+3, \lambda+6}-\frac{2 \lambda+1}{2} t_{\lambda, \lambda+4} t_{\lambda+4, \lambda+6}, \\
& \omega_{\lambda, \lambda+7}(t)=\left\{\begin{array}{cc}
-\frac{c}{b} t_{\lambda, \lambda+4} t_{\lambda+4, \lambda+7}, & \text { if } \quad b \neq 0 \\
-c t_{\lambda, \lambda+3} t_{\lambda+3, \lambda+7}, & \text { if } \quad b=0
\end{array}, \text { if } \lambda \notin\{-6,0\},\right. \\
& \omega_{0,7}(t)=-\frac{1}{7}\left(\frac{11}{10} t_{0,3} t_{3,7}+\frac{1}{2} t_{0,4} t_{4,7}+3 t_{0,5} t_{5,7}\right), \\
& \omega_{-6,1}(t)=\frac{1}{14}\left(t_{-6,-3} t_{-3,1}-6 t_{-6,-4} t_{-4,1}+\frac{11}{5} t_{-6,-2} t_{-2,1}\right), \\
& \omega_{0,8}(t)=\frac{2}{11} t_{0,5} t_{5,8} \\
& \omega_{-7,1}(t)=\frac{2}{15} t_{-7,-4} t_{-4,1} .
\end{aligned}
$$

These functions $\omega_{\lambda, \lambda+k}(t), k=5,6,7$, will appear as coefficients for some maps from $\mathcal{F}_{\lambda}$ to $\mathcal{F}_{\lambda+k}$ and they will be used in the expressions of integrability conditions. More precisely, we will show that the second term $\mathcal{L}^{(2)}$ is of the form $\mathcal{L}^{(2)}=\sum_{\lambda, k} \omega_{\lambda, \lambda+k}(t) J_{k+1}^{-1, \lambda}$.

Proposition 7. For $k=5,6,7$, we have the following second-order integrability conditions of the infinitesimal deformation (9)

$$
\begin{array}{ll}
\omega_{\lambda, \lambda+5}(t)=0 & \text { if } \lambda \in\{0,-2,-4\}, \\
\omega_{\lambda, \lambda+6}(t)=0 & \text { if } \lambda \in\left\{-\frac{5 \pm \sqrt{19}}{2},-\frac{5}{2}\right\}, \\
b t_{\lambda, \lambda+3} t_{\lambda+3, \lambda+7}-a t_{\lambda, \lambda+4} t_{\lambda+4, \lambda+7}=0 & \text { if } \lambda \notin\{0,-2,-4,-6\}, \\
10 t_{-2,0} t_{0,5}-t_{-2,1} t_{1,5}-\frac{1}{3} t_{-2,2} t_{2,5}=0, & \\
10 t_{-4,1} t_{1,3}+t_{-4,-1} t_{-1,3}+3 t_{-4,0} t_{0,3}=0 . &
\end{array}
$$

where $a$ and $b$ are defined by (8).

Proof. 1) For $k=5$, we have

$$
B_{\lambda, \lambda+5}=-t_{\lambda, \lambda+2} t_{\lambda+2, \lambda+5} \llbracket C_{\lambda+2, \lambda+5}, C_{\lambda, \lambda+2} \rrbracket-t_{\lambda, \lambda+3} t_{\lambda+3, \lambda+5} \llbracket C_{\lambda+3, \lambda+5}, C_{\lambda, \lambda+5} \rrbracket,
$$

hence, according to Proposition 3, we have

$$
B_{\lambda, \lambda+5}=\omega_{\lambda, \lambda+5}(t) \Omega_{\lambda, \lambda+5} .
$$

Thus, by Proposition 3, the $\mathfrak{s l}(2)$-relative 2 -cocycle $\Omega_{\lambda, \lambda+5}$ is nontrivial if and only if $\lambda \in$ $\{0,-2,-4\}$. Hence, for $\lambda \in\{0,-2,-4\}$, the condition $\omega_{\lambda, \lambda+5}(t)=0$ holds.

2) For $k=6$, as before, we have

$$
B_{\lambda, \lambda+6}=\omega_{\lambda, \lambda+6}(t) \Omega_{\lambda, \lambda+6} .
$$

Thus, if $\lambda \in\left\{-\frac{5 \pm \sqrt{19}}{2},-\frac{5}{2}\right\}$ the condition $\omega_{\lambda, \lambda+6}(t)=0$ must be satisfied. 
$3)$ Let $k=7$. Note that, hereafter, some singular values of the parameter $\lambda$ appear because the $\mathfrak{s l}(2)$-relative 2-cocycles $C_{\lambda, \lambda+5}$ exist only for $\lambda=0,-4$ and $C_{\lambda, \lambda+6}$ exist only for $\lambda=-\frac{5 \pm \sqrt{19}}{2}$.

i) For $\lambda \notin\{0,-2,-4,-6\}$, we have

$$
B_{\lambda, \lambda+7}=-t_{\lambda, \lambda+3} t_{\lambda+3, \lambda+7} \Omega_{\lambda, \lambda+7}-t_{\lambda, \lambda+4} t_{\lambda+4, \lambda+7} \widetilde{\Omega}_{\lambda, \lambda+7} .
$$

Therefore, the following conditions follow from Proposition 4

$$
b t_{\lambda, \lambda+3} t_{\lambda+3, \lambda+7}-a t_{\lambda, \lambda+4} t_{\lambda+4, \lambda+7}=0 .
$$

Indeed, according to the equation (8), if $b \neq 0$, we have

$$
B_{\lambda, \lambda+7}=-\frac{1}{b}\left(b t_{\lambda+3, \lambda+7}-a t_{\lambda, \lambda+4} t_{\lambda+4, \lambda+7}\right) \Omega_{\lambda, \lambda+7}+\omega_{\lambda, \lambda+7}(t) \partial J_{8}^{-1, \lambda}
$$

and if $b=0$, we have

$$
B_{\lambda, \lambda+7}=-t_{\lambda, \lambda+4} t_{\lambda+4, \lambda+7} \widetilde{\Omega}_{\lambda, \lambda+7}+\omega_{\lambda, \lambda+7}(t) \partial J_{8}^{-1, \lambda} .
$$

ii) By a direct computation, we show that

$$
B_{0,7}=\omega_{0,7}(t) \partial J_{8}^{-1,0} \quad \text { and } \quad B_{-6,1}=\omega_{-6,1}(t) \partial J_{8}^{-1,-6} .
$$

Hence, there are no conditions on $B_{0,7}$ and $B_{-6,1}$.

iii) For the other two singular values of $\lambda$ we have

$$
\begin{aligned}
& B_{-2,5}=-t_{-2,0} t_{0,5} \llbracket C_{0,5}, C_{-2,0} \rrbracket-t_{-2,1} t_{1,5} \Omega_{-2,5}-t_{-2,2} t_{2,5} \widetilde{\Omega}_{-2,5}, \\
& B_{-4,3}=-t_{-4,1} t_{1,3} \llbracket C_{1,3}, C_{-4,1} \rrbracket-t_{-4,-1} t_{-1,3} \Omega_{-4,3}-t_{-4,0} t_{0,3} \widetilde{\Omega}_{-4,3} .
\end{aligned}
$$

More precisely, we get

$$
\begin{aligned}
& B_{-2,5}=\left(10 t_{-2,0} t_{0,5}-t_{-2,1} t_{1,5}-\frac{1}{3} t_{-2,2} t_{2,5}\right) \Omega_{-2,5}+\omega_{-2,5}(t) \partial J_{8}^{-1,-2}, \\
& B_{-4,3}=-\left(10 t_{-4,1} t_{1,3}+t_{-4,-1} t_{-1,3}+3 t_{-4,0} t_{0,3}\right) \Omega_{-4,3}+\omega_{-4,3}(t) \partial J_{8}^{-1,-4} .
\end{aligned}
$$

So, the following integrability conditions become again from Proposition 4:

$$
10 t_{-2,0} t_{0,5}-t_{-2,1} t_{1,5}-\underline{1}_{-2,2} t_{2,5}=10 t_{-4,1} t_{1,3}+t_{-4,-1} t_{-1,3}+3 t_{-4,0} t_{0,3}=0 .
$$

Proposition 8. For $k=8,9,10$, we have the following second-order integrability conditions of the infinitesimal deformation (9), where in the first line $\lambda \notin\{0,-3,-4,-7\}$,

$$
\begin{aligned}
& t_{\lambda, \lambda+4} t_{\lambda+4, \lambda+8}=11 t_{0,4} t_{4,8}+10 t_{0,5} t_{5,8}=0, \\
& t_{-3,1} t_{1,5}-10 t_{-3,0} t_{0,5}=t_{-4,0} t_{0,4}+10 t_{-4,1} t_{1,4}=0, \\
& 11 t_{-7,-3} t_{-3,1}-10 t_{-7,-4} t_{-4,1}=t_{a_{i}, a_{i}+6} t_{a_{i}+6, a_{i}+8}=0, \\
& t_{a_{i}-2, a_{i}} t_{a_{i}, a_{i}+6}=t_{0,5} t_{5,9}=0, \\
& t_{-4,0} t_{0,5}-t_{-4,1} t_{1,5}=t_{-8,-4} t_{-4,1}=0, \\
& t_{a_{i}, a_{i}+6} t_{a_{i}+6, a_{i}+9}=t_{a_{i}-3, a_{i}} t_{a_{i}, a_{i}+6}=0, \\
& t_{a_{i}, a_{i}+6} t_{a_{i}+6, a_{i}+10}=t_{a_{i}-4, a_{i}} t_{a_{i}, a_{i}+6}=0 .
\end{aligned}
$$

Proof. 1) For $k=8$, we first recall that the cup-product $\Omega_{\lambda, \lambda+8}=\llbracket C_{\lambda, \lambda+4}, C_{\lambda+4, \lambda+8} \rrbracket$ is a $\mathfrak{s l}(2)$ relative nontrivial 2-cocycle. Moreover, for $\lambda \notin\left\{0,-3,-4,-7, a_{1}, a_{2}, a_{1}-2, a_{2}-2\right\}$, we have

$$
B_{\lambda, \lambda+8}=-t_{\lambda, \lambda+4} t_{\lambda+4, \lambda+8} \Omega_{\lambda, \lambda+8} .
$$


For the singular values, we easily check that

$$
\begin{array}{ll}
\llbracket C_{5,8}, C_{0,5} \rrbracket=\frac{10}{11} \Omega_{0,8}+\frac{2}{11} \partial J_{9}^{-1,0}, & \llbracket C_{0,5}, C_{-3,0} \rrbracket=10 \Omega_{-3,5}, \\
\llbracket C_{-4,1}, C_{-7,-4} \rrbracket=\frac{10}{11} \Omega_{-7,1}+\frac{2}{15} \partial J_{9}^{-1,-7}, & \llbracket C_{1,4}, C_{-4,1} \rrbracket=-10 \Omega_{-4,4}
\end{array}
$$

and we show that $\llbracket C_{a_{i}+6, a_{i}+8}, C_{a_{i}, a_{i}+6} \rrbracket$, and $\llbracket C_{a_{i}, a_{i}+6}, C_{a_{i}-2, a_{i}} \rrbracket$ are also nontrivial 2-cocycles.

Thus, we deduce all integrability conditions corresponding to the case $k=8$.

2) For $k=9$, the integrability conditions follow from the fact that any corresponding cupproduct of 1-cocycle is nontrivial. Moreover, we have only singular cases and we also show that $\llbracket C_{1,5}, C_{-4,1} \rrbracket=-\llbracket C_{0,5}, C_{-4,0} \rrbracket$.

3) For $k=10$ and $\lambda \neq a_{i}, a_{i}-4$ we have $B_{\lambda, \lambda+10}=0$. For $\lambda=a_{i}, a_{i}-4$ we have

$$
\begin{aligned}
& B_{a_{i}, a_{i}+10}=-t_{a_{i}, a_{i}+6} t_{a_{i}+6, a_{i}+10} \llbracket C_{a_{i}, a_{i}+6}, C_{a_{i}+6, a_{i}+10} \rrbracket=-t_{a_{i}, a_{i}+6} t_{a_{i}+6, a_{i}+10} \Omega_{a_{i}, a_{i}+10}, \\
& B_{a_{i}-4, a_{i}+6}=-t_{a_{i}-4, a_{i}} t_{a_{i}, a_{i}+6} \llbracket C_{a_{i}-4, a_{i}}, C_{a_{i}, a_{i}+6} \rrbracket=-t_{a_{i}-4, a_{i}} t_{a_{i}, a_{i}+6} \Omega_{a_{i}-4, a_{i}+6} .
\end{aligned}
$$

Like in the previous case we prove that the 2-cocycles $\Omega_{a_{i}, a_{i}+10}$ and $\Omega_{a_{i}-4, a_{i}+6}$ are nontrivial and then we deduce the corresponding integrability conditions.

Our main result in this section is the following

Theorem 2. The conditions (17) and (18) are necessary and sufficient for second-order integrability of the $\mathfrak{s l}(2)$-trivial infinitesimal deformation (9).

Proof. Of course, these conditions are necessary as, it was shown in Proposition 7 and Proposition 8. Now, under these conditions, the second term $\mathcal{L}^{(2)}$ of the the $\mathfrak{s l}(2)$-trivial infinitesimal deformation (9) is a solution of the Maurer-Cartan equation (16). This solution is defined up to a 1-coboundary and it has been shown in [7,2] that different choices of solutions of the Maurer-Cartan equation correspond to equivalent deformations. Thus, we can always choose

$$
\begin{aligned}
\mathcal{L}^{(2)}= & \frac{1}{2} \sum_{\lambda \neq 0,-2,-4} \omega_{\lambda, \lambda+5}(t) J_{6}^{-1, \lambda}+\frac{1}{2} \sum_{\lambda \neq a_{i},-\frac{5}{2}} \omega_{\lambda, \lambda+6}(t) J_{7}^{-1, \lambda} \\
& +\frac{1}{2} \sum_{\lambda} \omega_{\lambda, \lambda+7}(t) J_{8}^{-1, \lambda}+\frac{1}{2} \sum_{\lambda=0,-7} \omega_{\lambda, \lambda+8}(t) J_{9}^{-1, \lambda} .
\end{aligned}
$$

Of course, any $t_{\lambda, \lambda+k}$ appears in the expressions of $\mathcal{L}^{(1)}$ or $\mathcal{L}^{(2)}$ if and only if $\delta-\lambda$ and $k$ are integers satisfying $\delta-n \leq \lambda, \lambda+k \leq \delta$. Theorem 2 is proved.

\section{Third and fourth-order integrability conditions}

\subsection{Computing the third-order Maurer-Cartan equation}

Now we reconsider the formal deformation (11) which is a formal power series in the parameters $t_{\lambda, \lambda+j}$ with coefficients in $\mathcal{D}$. We suppose that the second-order integrability conditions are satisfied. So, the third-order terms of (11) are solutions of the (Maurer-Cartan) equation

$$
\partial \mathcal{L}^{(3)}=-\frac{1}{2} \sum_{i+j=3} \llbracket \mathcal{L}^{(i)}, \mathcal{L}^{(j)} \rrbracket
$$

As in the previous section we can write

$$
\partial \mathcal{L}^{(3)}=-\frac{1}{2} \sum_{k, \lambda} E_{\lambda, \lambda+k},
$$


where $E_{\lambda, \lambda+k}$ are maps from $\operatorname{Vect}_{\mathrm{Pol}}(\mathbb{R}) \times \operatorname{Vect}_{\mathrm{Pol}}(\mathbb{R})$ to $\mathcal{D}_{\lambda, \lambda+k}$. The third-order term $\mathcal{L}^{(3)}$ of the $\mathfrak{s l}(2)$-trivial formal deformation (11) is a solution of (20). So, the 2-cochains $E_{\lambda, k}$ must satisfy $E_{\lambda, k}=\partial J_{k+1}^{-1, \lambda}$ and then the third-order integrability conditions are deduced from this fact.

It is easy to see that $E_{\lambda, \lambda+k}=0$ for $k \leq 6$ or $k \geq 13$, so we compute successively the $E_{\lambda, \lambda+k}$ for $k=7, \ldots, 12$ and we resolve $E_{\lambda, \lambda+k}=\partial J_{k+1}^{-1, \lambda}$ to get the corresponding third-order integrability conditions.

Here, we mention that the maps $E_{\lambda, \lambda+k}$ are 2-cochains, but they are not necessarily 2cocycles because they are not cup-products of 1-cocycles like the maps $B_{\lambda, \lambda+k}$. Indeed, $\mathcal{L}^{(2)}$ is not necessarily a 1-cocycle.

\subsection{Third-order integrability conditions}

Proposition 9. For $k=7,8$, we have the following third-order integrability conditions of the infinitesimal deformation (9), for all $\lambda$

$$
\begin{aligned}
& t_{\lambda, \lambda+2} \omega_{\lambda+2, \lambda+7}(t)=\omega_{\lambda, \lambda+5}(t) t_{\lambda+5, \lambda+7}=0 \\
& t_{\lambda, \lambda+2} \omega_{\lambda+2, \lambda+8}(t)=\omega_{\lambda, \lambda+6}(t) t_{\lambda+6, \lambda+8}=0, \\
& t_{\lambda, \lambda+3} \omega_{\lambda+3, \lambda+8}(t)=\omega_{\lambda, \lambda+5}(t) t_{\lambda+5, \lambda+8}=0 .
\end{aligned}
$$

Proof. For $k=7$ and $\lambda \notin\{0,-2,-4,-6\}$ we have

$$
\begin{aligned}
& E_{\lambda, \lambda+7}=t_{\lambda, \lambda+2} \omega_{\lambda+2, \lambda+7}(t) \llbracket J_{6}^{-1, \lambda+2}, C_{\lambda, \lambda+2} \rrbracket+t_{\lambda+5, \lambda+7} \omega_{\lambda, \lambda+5}(t) \llbracket C_{\lambda+5, \lambda+7}, J_{6}^{-1, \lambda} \rrbracket, \\
& E_{0,7}=t_{0,2} \omega_{2,7}(t) \llbracket J_{6}^{-1,2}, C_{0,2} \rrbracket, \quad E_{-6,1}=t_{-1,1} \omega_{-6,-1}(t) \llbracket C_{-1,1}, J_{6}^{-1,-6} \rrbracket
\end{aligned}
$$

and

$$
E_{-2,5}=E_{-4,3}=0
$$

By a direct computation, we show that the three maps $\llbracket J_{6}^{-1, \lambda+2}, C_{\lambda, \lambda+2} \rrbracket, \llbracket C_{\lambda+5, \lambda+7}, J_{6}^{-1, \lambda} \rrbracket$ and $\partial J_{8}^{-1, \lambda}$ are linearly independent, for all $\lambda$. Thus,

$$
\begin{array}{ll}
t_{\lambda, \lambda+2} \omega_{\lambda+2, \lambda+7}(t)=0 & \text { for } \quad \lambda \neq-2,-4,-6, \\
t_{\lambda+5, \lambda+7} \omega_{\lambda, \lambda+5}(t)=0 & \text { for } \quad \lambda \neq 0,-2,-4 .
\end{array}
$$

But, under the second-order integrability conditions: $\omega_{\lambda, \lambda+5}(t)=0$ for $\lambda \in\{0,-2,-4\}$, the conditions

$$
t_{\lambda, \lambda+2} \omega_{\lambda+2, \lambda+7}(t)=\omega_{\lambda, \lambda+5}(t) t_{\lambda+5, \lambda+7}=0
$$

hold for all $\lambda$.

Now, for $k=8$ and $\lambda \notin\left\{a_{1}, a_{2},-\frac{5}{2}, a_{1}-2, a_{2}-2,-\frac{9}{2}, 0,-2,-4,-3,-5,-7\right\}$ we have

$$
\begin{aligned}
E_{\lambda, \lambda+8}= & t_{\lambda, \lambda+2} \omega_{\lambda+2, \lambda+8}(t) \llbracket J_{7}^{-1, \lambda+2}, C_{\lambda, \lambda+2} \rrbracket+t_{\lambda+6, \lambda+8} \omega_{\lambda, \lambda+6}(t) \llbracket C_{\lambda+6, \lambda+8}, J_{7}^{-1, \lambda} \rrbracket \\
& +t_{\lambda, \lambda+3} \omega_{\lambda+3, \lambda+8}(t) \llbracket J_{6}^{-1, \lambda+3}, C_{\lambda, \lambda+3} \rrbracket+t_{\lambda+5, \lambda+8} \omega_{\lambda, \lambda+5}(t) \llbracket C_{\lambda+5, \lambda+8}, J_{6}^{-1, \lambda} \rrbracket .
\end{aligned}
$$

As before, we show that

$$
t_{\lambda, \lambda+2} \omega_{\lambda+2, \lambda+8}(t)=t_{\lambda, \lambda+3} \omega_{\lambda+3, \lambda+8}(t)=t_{\lambda+5, \lambda+8} \omega_{\lambda, \lambda+5}(t)=t_{\lambda+6, \lambda+8} \omega_{\lambda, \lambda+6}(t)=0 .
$$

We get the same results for $\lambda \in\left\{a_{1}, a_{2},-\frac{5}{2}, a_{1}-2, a_{2}-2,-\frac{9}{2}, 0,-2,-4,-3,-5,-7\right\}$ by considering the second-order integrability conditions. 
Proposition 10. For $k=9$, we have the following third-order integrability conditions of the infinitesimal deformation (9), for all $\lambda$ :

$$
\begin{aligned}
& t_{\lambda, \lambda+3} \omega_{\lambda+3, \lambda+9}(t)=\omega_{\lambda, \lambda+6}(t) t_{\lambda+6, \lambda+9}=0, \\
& t_{\lambda, \lambda+4} \omega_{\lambda+4, \lambda+9}(t)=\omega_{\lambda, \lambda+5}(t) t_{\lambda+5, \lambda+9}=0, \\
& t_{\lambda-2, \lambda} \omega_{\lambda, \lambda+7}(t)=\omega_{\lambda, \lambda+7}(t) t_{\lambda+7, \lambda+9}=0 .
\end{aligned}
$$

Proof. For $k=9$ and $\lambda \notin\left\{0,-2,-4,-6,-8, a_{i},-\frac{5}{2}, a_{i}-3,-\frac{11}{2}\right\}$ we have

$$
\begin{aligned}
E_{\lambda, \lambda+9}= & t_{\lambda, \lambda+3} \omega_{\lambda+3, \lambda+9}(t) \llbracket J_{7}^{-1, \lambda+3}, C_{\lambda, \lambda+3} \rrbracket+t_{\lambda, \lambda+4} \omega_{\lambda+4, \lambda+9}(t) \llbracket J_{6}^{-1, \lambda+4}, C_{\lambda, \lambda+4} \rrbracket \\
& +t_{\lambda+5, \lambda+9} \omega_{\lambda, \lambda+5}(t) \llbracket C_{\lambda+5, \lambda+9}, J_{6}^{-1, \lambda} \rrbracket+t_{\lambda+6, \lambda+9} \omega_{\lambda, \lambda+6}(t) \llbracket C_{\lambda+6, \lambda+9}, J_{7}^{-1, \lambda} \rrbracket .
\end{aligned}
$$

The equation $E_{\lambda, \lambda+9}=\partial J_{10}^{-1, \lambda}$ gives

$$
t_{\lambda, \lambda+3} \omega_{\lambda+3, \lambda+9}(t)=t_{\lambda, \lambda+4} \omega_{\lambda+4, \lambda+9}(t)=t_{\lambda+5, \lambda+9} \omega_{\lambda, \lambda+5}(t)=t_{\lambda+6, \lambda+9} \omega_{\lambda, \lambda+6}(t)=0 .
$$

By considering the second-order integrability conditions, we get the same results for each $\lambda \in$ $\left\{0,-2,-4,-6,-8, a_{i},-\frac{5}{2}, a_{i}-3,-\frac{11}{2}\right\}$.

Proposition 11. For $k=10$, we have the following third-order integrability conditions of the infinitesimal deformation (9)

$$
\begin{array}{ll}
t_{\lambda-2, \lambda} \omega_{\lambda, \lambda+8}(t)=\omega_{\lambda, \lambda+8}(t) t_{\lambda+8, \lambda+10}=0 & \text { for } \lambda=0,-7, \\
t_{\lambda-3, \lambda} \omega_{\lambda, \lambda+7}(t)=\omega_{\lambda, \lambda+7}(t) t_{\lambda+7, \lambda+10}=0 & \text { for all } \lambda, \\
t_{\lambda, \lambda+4} \omega_{\lambda+4, \lambda+10}(t)=\omega_{\lambda, \lambda+6}(t) t_{\lambda+6, \lambda+10}=0 & \text { for all } \lambda, \\
t_{\lambda, \lambda+5} \omega_{\lambda+5, \lambda+10}(t)=\omega_{\lambda-5, \lambda}(t) t_{\lambda, \lambda+5}=0 & \text { for } \lambda=0,-4 .
\end{array}
$$

Proof. For $k=10$ and $\lambda \notin\left\{-9,-7,-\frac{13}{2},-6,-5,-4,-3,-2,-\frac{5}{2}, 0, a_{1}, a_{1}-4, a_{2}, a_{2}-4\right\}$ we have

$$
E_{\lambda, \lambda+10}=t_{\lambda, \lambda+4} \omega_{\lambda+4, \lambda+10}(t) \llbracket J_{7}^{-1, \lambda+4}, C_{\lambda, \lambda+4} \rrbracket+t_{\lambda+6, \lambda+10} \omega_{\lambda, \lambda+6}(t) \llbracket C_{\lambda+6, \lambda+10}, J_{7}^{-1, \lambda} \rrbracket .
$$

The equation $E_{\lambda, \lambda+10}=\partial J_{11}^{-1, \lambda}$ gives the conditions

$$
t_{\lambda, \lambda+4} \omega_{\lambda+4, \lambda+10}(t)=t_{\lambda+6, \lambda+10} \omega_{\lambda, \lambda+6}(t)=0 .
$$

We check that, for $\lambda \in\left\{-9,-7,-\frac{13}{2},-6,-5,-4,-3,-2,-\frac{5}{2}, 0, a_{1}, a_{1}-4, a_{2}, a_{2}-4\right\}$, these latter conditions must be also satisfied. The others conditions follow from the singular values of $\lambda$.

Proposition 12. For $k=11$, we have the following second-order integrability conditions of the infinitesimal deformation (9)

$$
\begin{array}{ll}
t_{\lambda, \lambda+6} \omega_{\lambda+6, \lambda+11}(t)=\omega_{\lambda-5, \lambda}(t) t_{\lambda, \lambda+6}=0 & \text { for } \lambda=-\frac{5 \pm \sqrt{19}}{2}, \\
t_{\lambda, \lambda+5} \omega_{\lambda+5, \lambda+11}(t)=\omega_{\lambda-6, \lambda}(t) t_{\lambda, \lambda+5}=0 & \text { for } \lambda=0,-4, \\
t_{\lambda-4, \lambda} \omega_{\lambda, \lambda+7}(t)=\omega_{\lambda, \lambda+7}(t) t_{\lambda+7, \lambda+11}=0 & \text { for all } \lambda, \\
t_{\lambda-3, \lambda} \omega_{\lambda, \lambda+8}(t)=\omega_{\lambda, \lambda+8}(t) t_{\lambda+8, \lambda+11}=0 & \text { for } \lambda=0,-7 .
\end{array}
$$

Proposition 13. For $k=12$, we have the following third-order integrability conditions of the infinitesimal deformation (9)

$$
\begin{array}{ll}
t_{\lambda, \lambda+5} \omega_{\lambda+5, \lambda+12}(t)=\omega_{\lambda-7, \lambda}(t) t_{\lambda, \lambda+5}=0, & \text { for } \quad \lambda=0,-4, \\
t_{\lambda, \lambda+6} \omega_{\lambda+6, \lambda+12}(t)=\omega_{\lambda-6, \lambda}(t) t_{\lambda, \lambda+6}=0 & \text { for } \quad \lambda=-\frac{5 \pm \sqrt{19}}{2}, \\
t_{\lambda-4, \lambda} \omega_{\lambda, \lambda+8}(t)=\omega_{\lambda, \lambda+8}(t) t_{\lambda+8, \lambda+12}=0 & \text { for } \quad \lambda=0,-7 .
\end{array}
$$

Proof. For $k=11$ and $k=12$, the 2-cochains $E_{\lambda, \lambda+k}$ are defined only for some particular values of $\lambda$. We compute these 2-cochains and then we check the corresponding integrability conditions. 


\subsection{Fourth-order integrability conditions}

Proposition 14. For generic $\lambda$, the fourth-order integrability conditions of the infinitesimal deformation (9) are the following:

$$
\omega_{\lambda, \lambda+i}(t) \omega_{\lambda+i, \lambda+k}(t)=0, \quad \text { where } \quad 5 \leq i \leq 7 \quad \text { and } \quad 5+i \leq k \leq 7+i .
$$

Proof. These conditions come from the fact that the fourth term $\mathcal{L}^{(4)}$ must satisfy:

$$
\partial \mathcal{L}^{(4)}=-\frac{1}{2} \llbracket \mathcal{L}^{(2)}, \mathcal{L}^{(2)} \rrbracket .
$$

Indeed, we can always reduce $\mathcal{L}^{(3)}$ to zero by equivalence.

The following theorem is our main result.

Theorem 3. The second-order integrability conditions (17) and (18) together with the third and the fourth-order conditions (21)-(26) are necessary and sufficient for the complete integrability of the infinitesimal deformation (9). Moreover, any formal $\mathfrak{s l}(2)$-trivial deformation of the Lie derivative $L_{X}$ on the space of symbols $\mathcal{S}_{\delta}^{n}$ is equivalent to a polynomial one of degree equal or less than 2.

Proof. Clearly, all these conditions are necessary. So, let us prove that they are also sufficient. As in the proof of Theorem 2, the solution $\mathcal{L}^{(3)}$ of the Maurer-Cartan equation (19) is defined up to a 1-coboundary, thus, we can always reduce $\mathcal{L}^{(3)}$ to zero by equivalence. Moreover, by recurrence, the highest-order terms $\mathcal{L}^{(m)}$ satisfy the equation $\partial \mathcal{L}^{(m)}=0$ and can also be reduced to the identically zero map. This completes the proof of Theorem 3.

Remark 1. The majority of integrability conditions concern some parameters $t_{\lambda, \lambda+k}$ with singular values of $\lambda$. All these singular values of $\lambda$ are negatives. So, let us consider the space $\mathcal{S}_{\delta}^{n}$ with generic $\delta$, for example, $\delta-n>0$. In this case, the second-order integrability conditions are reduced to the following equations:

$$
b t_{\lambda, \lambda+3} t_{\lambda+3, \lambda+7}-a t_{\lambda, \lambda+4} t_{\lambda+4, \lambda+7}=t_{\lambda, \lambda+4} t_{\lambda+4, \lambda+8}=0 .
$$

\section{$7 \quad$ Examples}

Example 1. Let us consider the space of symbols $\mathcal{S}_{\lambda+4}^{4}$.

Proposition 15. Any formal $\mathfrak{s l}(2)$-trivial deformation of the $\operatorname{Vect}_{\mathrm{Pol}}(\mathbb{R})$-action on the space $\mathcal{S}_{\lambda+4}^{4}$ is equivalent to his infinitesimal part, without any conditions on the parameters (independent parameters). That is, the miniversal deformation here has base $\mathbb{C}[[t]]$ where $t$ designates the family of all parameters.

Proof. The infinitesimal $\mathfrak{s l}(2)$-trivial deformation, in this case, is given by

$$
\mathcal{L}=L+\mathcal{L}^{(1)},
$$

where $L_{X}$ is the Lie derivative of $\mathcal{S}_{\lambda+4}^{4}$ along the vector field $X \frac{d}{d x}$ defined by (1), and

$$
\begin{aligned}
\mathcal{L}^{(1)}= & t_{\lambda, \lambda+2} C_{\lambda, \lambda+2}+t_{\lambda, \lambda+3} C_{\lambda, \lambda+3}+t_{\lambda, \lambda+4} C_{\lambda, \lambda+4} \\
& +t_{\lambda+1, \lambda+3} C_{\lambda+1, \lambda+3}(X)+t_{\lambda+1, \lambda+4} C_{\lambda+1, \lambda+4}+t_{\lambda+2, \lambda+4} C_{\lambda+2, \lambda+4} .
\end{aligned}
$$

There are no conditions to integrate this infinitesimal deformation to a formal one. The solution $\mathcal{L}^{(2)}$ of (13) is defined up to a 1-coboundary and different choices of solutions of the MaurerCartan equation correspond to equivalent deformations. Thus, we can always reduce $\mathcal{L}^{(2)}$ to zero by equivalence. Then, by recurrence, the highest-order terms $\mathcal{L}^{(m)}$ satisfy the equation $\partial \mathcal{L}^{(m)}=0$ and $\mathcal{L}^{(m)}$ can also be reduced to the identically zero map. 
Remark 2. We have the same results for $\mathcal{S}_{\lambda+k}^{k}$ if $k \leq 4$. Indeed, for $k \leq 4$ there are no integrability conditions.

Example 2. Let us consider the $\operatorname{Vect}_{\mathrm{Pol}}(\mathbb{R})$-module $\mathcal{S}_{7}^{6}$.

Proposition 16. Any formal $\mathfrak{s l}(2)$-trivial deformation of the $\operatorname{Vect}_{\mathrm{Pol}}(\mathbb{R})$-action on the space $\mathcal{S}_{7}^{6}$ is equivalent to

$$
\mathcal{L}_{X}=L+\mathcal{L}^{(1)}+\mathcal{L}^{(2)}
$$

where

$$
\begin{aligned}
\mathcal{L}^{(1)}= & t_{1,3} C_{1,3}+t_{1,4} C_{1,4}+t_{1,5} C_{1,5}+t_{2,4} C_{2,4}+t_{2,5} C_{2,5}+t_{2,6} C_{2,6} \\
& +t_{3,5} C_{3,5}+t_{3,6} C_{3,6}+t_{3,7} C_{3,7}+t_{4,6} C_{4,6}+t_{4,7} C_{4,7}
\end{aligned}
$$

and

$$
\mathcal{L}^{(2)}=\frac{1}{2} \omega_{1,6}(t) J_{6}^{-1,1}+\frac{1}{2} \omega_{2,7}(t) J_{6}^{-1,2}+\frac{1}{2} \omega_{1,7}(t) J_{7}^{-1,1} .
$$

The formal deformation (27) is defined without any condition on the parameters (independent parameters). That is, the miniversal deformation here has base $\mathbb{C}[[t]]$ where $t$ designates the family of all parameters.

For $k \leq 6$, generically there are no integrability conditions which is the case of the previous example (see Remark 1). Now, we study a generic example with $k=7$.

Example 3. Let us consider the $\operatorname{Vect}_{\mathrm{Pol}}(\mathbb{R})$-module $\mathcal{S}_{\lambda+7}^{7}$ for generic $\lambda$.

Proposition 17. The Vect $\operatorname{Pol}_{\mathrm{P}}(\mathbb{R})$-module $\mathcal{S}_{\lambda+7}^{7}$ admits six $\mathfrak{s l}(2)$-trivial deformations with 11 independent parameters. It admits a miniversal $\mathfrak{s l}(2)$-trivial deformation with 15 parameters. These deformations are polynomial of degree 2 .

Proof. Any formal $\mathfrak{s l}(2)$-trivial deformation of the $\operatorname{Vect}_{\mathrm{Pol}}(\mathbb{R})$-action on the space $\mathcal{S}_{\lambda+7}^{7}$ is equivalent to

$$
\mathcal{L}=L+\mathcal{L}^{(1)}+\mathcal{L}^{(2)}
$$

where

$$
\begin{aligned}
\mathcal{L}^{(1)}= & t_{\lambda, \lambda+2} C_{\lambda, \lambda+2}+t_{\lambda, \lambda+3} C_{\lambda, \lambda+3}+t_{\lambda, \lambda+4} C_{\lambda, \lambda+4}+t_{\lambda+1, \lambda+3} C_{\lambda+1, \lambda+3} \\
& +t_{\lambda+1, \lambda+4} C_{\lambda+1, \lambda+4}+t_{\lambda+1, \lambda+5} C_{\lambda+1, \lambda+5}+t_{\lambda+2, \lambda+4} C_{\lambda+2, \lambda+4}+t_{\lambda+2, \lambda+5} C_{\lambda+2, \lambda+5} \\
& +t_{\lambda+2, \lambda+6} C_{\lambda+2, \lambda+6}+t_{\lambda+3, \lambda+5} C_{\lambda+3, \lambda+5}+t_{\lambda+3, \lambda+6} C_{\lambda+3, \lambda+6}+t_{\lambda+3, \lambda+7} C_{\lambda+3, \lambda+7} \\
& +t_{\lambda+4, \lambda+6} C_{\lambda+4, \lambda+6}+t_{\lambda+4, \lambda+7} C_{\lambda+4, \lambda+7}+t_{\lambda+5, \lambda+7} C_{\lambda+5, \lambda+7}
\end{aligned}
$$

and

$$
\begin{aligned}
\mathcal{L}^{(2)}= & \frac{1}{2} \omega_{\lambda, \lambda+5}(t) J_{6}^{-1, \lambda}+\frac{1}{2} \omega_{\lambda+1, \lambda+6}(t) J_{6}^{-1, \lambda+1}+\frac{1}{2} \omega_{\lambda+2, \lambda+7}(t) J_{6}^{-1, \lambda+2} \\
& +\frac{1}{2} \omega_{\lambda, \lambda+6}(t) J_{7}^{-1, \lambda}+\frac{1}{2} \omega_{\lambda+1, \lambda+7}(t) J_{7}^{-1, \lambda+1} .
\end{aligned}
$$

There are only three integrability conditions:

$$
b t_{\lambda, \lambda+3} t_{\lambda+3, \lambda+7}-a t_{\lambda, \lambda+4} t_{\lambda+4, \lambda+7}=t_{\lambda, \lambda+2} \omega_{\lambda+2, \lambda+7}(t)=\omega_{\lambda, \lambda+5}(t) t_{\lambda+5, \lambda+7}=0 .
$$

The formal $\mathfrak{s l}(2)$-trivial deformations with the greatest number of independent parameters are those corresponding to $b t_{\lambda, \lambda+3} t_{\lambda+3, \lambda+7}-a t_{\lambda, \lambda+4} t_{\lambda+4, \lambda+7}=t_{\lambda, \lambda+2}=t_{\lambda+5, \lambda+7}=0$. So, we must kill at least four parameters and there are six choices. Thus, there are four deformations with 11 independent parameters. Of course, there are many formal deformations with less then 11 independent parameters. The deformation $\mathcal{L}=L+\mathcal{L}^{(1)}+\mathcal{L}^{(2)}$, is the miniversal $\mathfrak{s l}(2)$ trivial deformation of $\mathcal{S}_{\lambda+7}^{7}$ with base $\mathcal{A}=\mathbb{C}[t] / \mathcal{R}$, where $t$ is the family of all parameters given in the expression of $\mathcal{L}^{(1)}$ and $\mathcal{R}$ is the ideal generated by the polynomials $b t_{\lambda, \lambda+3} t_{\lambda+3, \lambda+7}-$ $a t_{\lambda, \lambda+4} t_{\lambda+4, \lambda+7}$ and $\omega_{\lambda, \lambda+5}(t) t_{\lambda+5, \lambda+7}$. 


\section{Acknowledgements}

We would like to thank Sofiane Bouarroudj for his suggestions and for pointing out a mistake in a previous version of this paper.

\section{References}

[1] Agrebaoui B., Ben Fraj N., Ben Ammar M., Ovsienko V., Deformation of modules of differential forms, J. Nonlinear Math. Phys. 10 (2003), 148-156, math.QA/0310494.

[2] Agrebaoui B., Ammar F., Lecomte P., Ovsienko V., Multi-parameter deformations of the module of symbols of differential operators, Int. Math. Res. Not. 2002 (2002), no. 16, 847-869, math.QA/0011048.

[3] Bouarroudj S., On $\mathfrak{s l}(2)$-relative cohomology of the Lie algebra of vector fields and differential operators, J. Nonlinear Math. Phys. 14 (2007), 112-127, math.DG/0502372.

[4] Bouarroudj S., Ovsienko V., Three cocycles on $\operatorname{Diff}\left(S^{1}\right)$ generalizing the Schwarzian derivative, Int. Math. Res. Not. 1998 (1998), no. 1, 25-39, dg-ga/9710018.

[5] Fialowski A., Deformations of Lie algebras, Mat. Sb. 55 (1986), 467-473.

[6] Fialowski A., An example of formal deformations of Lie algebras, in Deformation Theory of Algebras and Structures and Applications, NATO Adv. Sci. Inst. Ser. C Math. Phys. Sci., Vol. 247, Kluwer Acad. Publ., Dordrecht, 1988, 375-401.

[7] Fialowski A., Fuchs D.B., Construction of miniversal deformations of Lie algebras, J. Funct. Anal. 161 (1999), 76-110, math.RT/0006117.

[8] Fuchs D.B., Cohomology of infinite-dimensional Lie algebras, Consultants Bureau, New York, 1987.

[9] Gargoubi H., Sur la géométrie de l'espace des opérateurs différentiels linéaires sur $\mathbb{R}$, Bull. Soc. Roy. Sci. Liège 69 (2000), 21-47.

[10] Gargoubi H., Mellouli N., Ovsienko V., Differential operators on supercircle: conformally equivariant quantization and symbol calculus, Lett. Math. Phys. 79 (2007), 51-65, math-ph/0610059.

[11] Gordan P., Invariantentheorie, Teubner, Leipzig, 1887.

[12] Nijenuis A., Richardson R.W. Jr., Deformations of homomorphisms of Lie groups and Lie algebras, Bull. Amer. Math. Soc. 73 (1967), 175-179.

[13] Ovsienko V., Roger C., Deforming the Lie algebra of vector fields on $S^{1}$ inside the Lie algebra of pseudodifferential operators on $S^{1}$, in Differential Topology, Infinite-Dimensional Lie Algebras, and Applications, Amer. Math. Soc. Transl. Ser. 2, Vol. 194, Amer. Math. Soc., Providence, RI, 1999, 211-226, math.QA/9812074.

[14] Ovsienko V., Roger C., Deforming the Lie algebra of vector fields on $S^{1}$ inside the Poisson algebra on $\dot{T}^{*} S^{1}$, Comm. Math. Phys. 198 (1998), 97-110, q-alg/9707007.

[15] Richardson R.W., Deformations of subalgebras of Lie algebras, J. Differential Geom. 3 (1969), $289-308$. 